\title{
Motivation prosociale et don de travail : une comparaison entre le secteur privé et la fonction publique d'État en France
}

Prosocial Motivation and Labour Donation: a Comparison between the Public and Private Sectors

\section{Priscilla Lemoyne}

\section{OpenEdition Journals}

Édition électronique

URL : http://journals.openedition.org/travailemploi/9284

DOI : 10.4000/travailemploi.9284

ISSN : $1775-416 \mathrm{X}$

\section{Éditeur}

DARES - Ministère du Travail

\section{Édition imprimée}

Date de publication : 2 novembre 2019

Pagination : $5-38$

ISSN : 0224-4365

\section{Référence électronique}

Priscilla Lemoyne, « Motivation prosociale et don de travail : une comparaison entre le secteur privé et la fonction publique d'État en France », Travail et Emploi [En ligne], 159 | 2019, mis en ligne le 01 décembre 2019, consulté le 07 décembre 2020. URL : http://journals.openedition.org/travailemploi/ 9284 ; DOI : https://doi.org/10.4000/travailemploi.9284 


\title{
Motivation prosociale et don de travail : une comparaison entre le secteur privé et la fonction publique d'État en France"
}

\author{
Priscilla Lemoyne**
}

\begin{abstract}
Par l'analyse du lien entre heures supplémentaires impayées et nature de l'employeur, nous cherchons à identifier l'existence d'une forme de motivation prosociale spécifique au secteur public. S'intéresser au comportement des agents du secteur public, fournisseurs de biens et services à caractère social et collectif, permet de tester la théorie du don de travail d'Anne E. PRESTON (1989). Nos résultats révèlent une absence de différence dans la fréquence d'offre d'heures supplémentaires non rémunérées entre les salariés des secteurs public et privé dans les données de l'enquête Changements organisationnels et informatisation (COI) 2006. Cependant, nous montrons que ces heures ne s'expliquent pas par les mêmes facteurs au sein des deux secteurs et qu'elles ne sont pas le produit des mêmes méthodes de management des ressources humaines. L'incitation à l'effort y prendrait des formes compatibles avec des sources de motivations de nature différente, ce qui invite à la prudence dans la mise en œuvre éventuelle des méthodes du nouveau management public, qui sont importées du secteur privé.
\end{abstract}

$\mathrm{D}$ ans de nombreuses enquêtes réalisées dans les pays européens, les salariés déclarent effectuer des heures supplémentaires non compensées, que ce soit par une rémunération financière ou par une déduction d'heures de travail. Ainsi, selon la confédération des syndicats britanniques, l'année 2016 est une année record avec 19,6\% des travailleurs affirmant accomplir des heures supplémentaires sans compensation, pour un supplément hebdomadaire de travail gratuit moyen de 7,7 heures.

\footnotetext{
* Ce travail a été réalisé au sein du Centre d'études de l'emploi et du travail (CEET) et du laboratoire Lemma (université Panthéon-Assas Paris 2). L'auteure tient à remercier Mahmood Arai, Nathalie Greenan, Sylvie Hamon-Cholet, Victor Hiller, Joseph Lanfranchi, Ali Skalli et Ioannis Theodossiou pour leur aide et leurs conseils précieux. Le texte final demeure bien évidemment de la seule responsabilité de l'auteure.

** Lemma EA 4442 (Laboratoire d'économie mathématique et de microéconomie appliquée), université PanthéonAssas ; Priscilla.lemoyne@ hotmail.fr.
} 
En Allemagne, le nombre d'heures non compensées a augmenté de $16 \%$ entre 1991 et 2014, au point de dépasser de près de $40 \%$ le nombre d'heures supplémentaires payées (WANGER et al., 2016). Pour la France, il ne semble pas exister de statistiques suivies de ce phénomène, mais une enquête menée par la Direction de l'animation de la recherche, des études et des statistiques (Dares) en 2010 a conclu que $14 \%$ des salariés travaillant à temps plein indiquaient travailler des heures supplémentaires non compensées, et pour trois quarts d'entre eux, chaque semaine (DEMOLY, 2011).

Ce constat statistique a fait l'objet de nombreux travaux économiques visant à expliquer un tel comportement qui ne cadre pas avec les hypothèses habituelles du modèle d'offre de travail, qui postule que les heures de travail sont pénibles, apportant une désutilité qui doit être compensée (ANGER, 2011 ; IOANNIDES et al., 2014 ; VAN Der Meer, Wielers, 2015 ; McKenzie, Rutherford, 2017). Dans ces travaux, ces efforts non rémunérés sont quasi systématiquement liés à des motivations extrinsèques à l'emploi (l'espoir d'une prime éventuelle ou l'amélioration des perspectives de promotion par exemple). Dans notre étude, nous considérons l'hypothèse selon laquelle l'existence d'une motivation intrinsèque ${ }^{1}$ chez les agents du secteur public inciterait ceux-ci à choisir volontairement d'offrir ces heures non compensées.

Ces heures pourraient en effet pour partie s'expliquer comme une forme de « don de travail », c'est-à-dire un effort supplémentaire fourni au-delà de ce qui est contractuellement nécessaire, en excluant toutes formes de motivations matérielles. La théorie du « don de travail » élaborée par Anne E. PRESTON (1989) explique les comportements des salariés en termes d'offre de travail en fonction de leurs motivations et montre que les salariés du secteur non lucratif acceptent de percevoir un salaire plus faible que celui qu'ils auraient pu toucher au sein du secteur privé à but lucratif, en raison de leur motivation prosociale à participer à la production de services à destination de la collectivité. En effet, par la nature de ces services, les travailleurs retirent une satisfaction directe et non pécuniaire à les fournir, car ils se préoccupent véritablement de leurs bénéficiaires (altruisme) et/ou parce qu'ils bénéficient d'une reconnaissance sociale. Cette motivation prosociale, par sa dimension altruiste, est une forme de motivation intrinsèque dans la mesure où le moteur qui conditionne l'action ne repose pas sur des récompenses monétaires ou matérielles.

Les sources de motivation des salariés sont par nature inobservables dans les statistiques. En revanche, la théorie des préférences révélées nous enseigne comment déduire de l'observation des comportements des individus leurs préférences et leurs motivations « individuelles », sous réserve de faire l'hypothèse de la rationalité de leurs décisions. Si l'engagement volontaire des salariés dans le secteur public plutôt que privé provient d'une motivation d'essence altruiste, nous devrions observer un comportement de don de travail plus fréquent de leur part.

1. Selon Edward DECI (1971, p. 105), « on dit qu'un individu est intrinsèquement motivé pour effectuer une tâche lorsqu'il n'en retire aucune récompense apparente, hormis l'activité en elle-même » (traduction de l'auteure). 
L'objectif de cette étude est donc d'utiliser les comportements observés des salariés en termes d'heures supplémentaires non compensées dans les secteurs public et privé afin de mettre en évidence l'existence de formes de motivations spécifiques dans chaque secteur. Nous compléterons ainsi l'étude de A. E. Preston (1989) en testant le bien-fondé de la théorie du « don de travail » dans le secteur public français.

La synthèse de la littérature sur les pratiques du management et sur les comportements des salariés des secteurs public et associatif en France fait ressortir la spécificité des formes de motivation qui y sont observées (MelniK, GuILlemot, 2010 ; NARCY, 2013a). Selon James L. PERrY (2000), les agents de l’État auraient choisi le secteur public parce qu'ils développeraient une motivation dite « de service public », c'est-àdire une prédisposition à participer aux missions spécifiques des institutions publiques. Pour Patrick FrANÇOIS et Michael VlassoPOULOS (2008), les salariés associatifs feraient montre d'une motivation prosociale expliquant leur engagement dans des organisations à but non lucratif qui se concentrent principalement dans des secteurs d'activité comme la santé, l'action sociale et l'éducation, dont les finalités sont de satisfaire l'intérêt général et de développer des actions de solidarité. Le secteur public dans son ensemble (qui inclut les collectivités locales et hôpitaux publics) étant davantage orienté vers des activités de production de biens et de services ayant pour principal objectif de satisfaire l'intérêt général, nous sommes amenées à supposer que les agents publics développent également une motivation prosociale au travail, différente de celle des salariés du privé.

Les motivations de service public et prosociale, proches par leur dimension altruiste, sont des formes de motivation intrinsèque, c'est-à-dire dont les leviers ne sont pas de nature matérielle ni ne relèvent d'une volonté de se forger une bonne réputation : elles résulteraient donc de la participation du salarié à une activité à laquelle il accorde une valeur per $\mathrm{se}^{2}$.

Deux formes d'explication justifient un don de travail dans le secteur public ou dans le secteur associatif. Selon la première, les individus intrinsèquement motivés pourraient s'engager dans un comportement prosocial dans n'importe quel environnement institutionnel, mais ils choisissent de travailler dans les secteurs public ou associatif du fait des missions rendues par ces secteurs. L'effet de sélection assurerait le lien entre le don de travail et l'institution employeuse (BESLEY, GHATAK, 2005). D'après la seconde, cette association entre don de travail et secteur institutionnel provient de ce que la non-recherche du profit par les secteurs public et associatif empêche que les bénéfices du don de travail de leurs salariés soient détournés dans une perspective de profit aux dépens de la recherche de la qualité du service. La forme institutionnelle de l'employeur permettrait ici de protéger le don de travail (FRANÇOIS, 2000).

2. La motivation de service public étant un cas particulier de motivation prosociale (GRANT, 2008 ; PERRY et al., 2010), nous choisissons d'utiliser l'expression « motivation prosociale » afin de désigner la motivation intrinsèque spécifique des agents de la fonction publique d'État (FPE). L'intention ici n'est pas d'identifier la forme exacte de la motivation qui anime les salariés, il est difficile en effet de distinguer si celle-ci provient davantage de la mission rendue par ce secteur et liée à la nature des biens et services distribués (santé, éducation, action sociale, etc.) ou si elle relève d'un engagement pour l'intérêt public. 
Peu d'analyses ont été menées sur la relation entre comportement prosocial et environnement institutionnel en utilisant des données issues des comportements d'effort sur le lieu de travail. Les trois études qui, à notre connaissance, correspondent à cette description se focalisent sur le comportement des salariés du secteur associatif. En France, Joseph LANFRANCHI et Mathieu NARCY (2006) ont étudié les différences de salaire et d'absence pour maladie entre les salariés des secteurs privés à but lucratif et à but non lucratif. Ils montrent que les salariés du secteur à but non lucratif perçoivent un salaire plus faible que celui qu'ils auraient pu obtenir dans le secteur privé à but lucratif et qu'en outre, ils s'absentent moins. Michail Veliziotis (2010) analyse pour la Grande-Bretagne l'effet d'appartenir à un syndicat sur les heures supplémentaires non payées et met en évidence que le fait d'être syndiqué n' augmente cette forme de don de travail que lorsque l'individu travaille dans un secteur fournissant des services sociaux ou non marchands. En Grande-Bretagne également, l'étude de Paul GrEGG et de ses co-auteurs (2011), qui mobilise les données du Panel britannique des ménages (British Household Panel Survey - BHPS), conclut que les salariés publics et associatifs, considérés ensemble, semblent consentir un don de travail en acceptant d'offrir des heures de travail dans une proportion supérieure à celle de leurs homologues du secteur privé à but lucratif.

Dans notre étude, nous utilisons une mesure de don de travail semblable à celle proposée par P. GREGG et ses co-auteurs (2011), en l'appliquant à l'analyse des comportements des agents publics français (fonctionnaires et contractuels) ${ }^{3}$ à partir des données de l'enquête Changements organisationnels et informatisation (COI) menée en 2006-2007. Ce dispositif présente l'avantage d'interroger à la fois des organisations du secteur privé et la fonction publique d'État (FPE). Il permet, d'une part, de disposer de données sur l'organisation du travail et de la production dans les organisations employeuses grâce aux questions posées aux employeurs des secteurs public et privé et, d'autre part, d'analyser le comportement ou le ressenti du vécu au travail des salariés échantillonnés grâce à un questionnaire identique pour les deux secteurs. COI présente en outre l'intérêt de s'être déroulé dans un contexte de réforme de l'État. En effet, la FPE a connu au début des années 2000 des changements de grande ampleur tant sur le plan de son organisation que des outils informatiques utilisés ${ }^{4}$. La mise en œuvre de nouveaux outils managériaux dans le domaine des ressources humaines, du pilotage des activités ou encore des relations avec les usagers a été pour partie impulsée par la loi organique relative aux lois de finance (Lolf), qui est progressivement entrée en vigueur de 2003 à $2006^{5}$. Grâce à COI, nous pouvons observer les comportements d'effort des travailleurs des deux secteurs à l'aune de ces évolutions.

3. Les travailleurs du secteur public considérés dans notre étude sont les agents de la fonction publique d'État, hors périmètre du ministère de la Défense, employés dans des directions d'administration centrale ou déconcentrée, des services déconcentrés, des académies ou des établissements scolaires et ne sont ni enseignants ni magistrats.

4. Selon COI, les changements informatiques et organisationnels sont de plus grande ampleur sur la période 2003-2006 dans la FPE que dans le secteur privé et ont des effets spécifiques sur la qualité de vie au travail selon les secteurs (GREENAN et al., 2016).

5. Toujours d'après Nathalie GreEnAN et ses co-auteurs (2016), la Lolf a été déterminante dans les changements radicaux advenus dans la FPE entre 2003 et 2006. 
Interroger les formes de motivation au travail selon le secteur employeur nous paraît particulièrement utile dans un contexte où l'efficience du secteur public a été remise en question à la fois du point de vue de sa gestion, ce qui se traduit par la mise en place d'une réorganisation profonde de ses méthodes de management, et de l'engagement de ses agents, souvent soupçonnés d'être des «tire-au-flanc », comme le montrent les débats autour du jour de carence des fonctionnaires. En ce sens, nos résultats ont des implications importantes en termes de management des ressources humaines. Dans un contexte d'homogénéisation des modes de management entre les secteurs public et privé, il est important de bien connaître la spécificité des formes de motivation existantes en leur sein, car elles n'appellent pas nécessairement les mêmes méthodes de gestion des ressources humaines.

Notre article est organisé comme suit. Dans une première partie, nous décrivons l'approche théorique du don de travail tout en proposant une revue de la littérature traitant des autres explications des heures supplémentaires non compensées. Puis nous proposons une description détaillée des données avant de présenter les différentes méthodologies économétriques utilisées et d'exposer nos résultats et commentaires.

\section{Les heures supplémentaires non payées : un don de travail ?}

La théorie économique traditionnelle de l'offre de travail stipule que la fonction d'utilité individuelle est une fonction décroissante du niveau d'effort au travail. Ainsi, de manière à accroître l'effort de ses salariés, une organisation peut utiliser des mécanismes incitatifs tels que les récompenses monétaires. Cependant, l'influence croissante des théories psychologiques de la motivation individuelle a engendré des travaux, théoriques comme empiriques, mettant l'accent sur le rôle joué par la motivation intrinsèque sur l'effort et la performance des travailleurs au sein des différentes organisations modernes de travail.

\section{La théorie du don de travail}

À l'origine, la théorie du don de travail de A. E. PRESTON (1989) vise à rationaliser les différences de rémunération entre les salariés selon la source de leurs motivations. Dans ce cadre, les salariés du secteur associatif, à but non lucratif, devraient, pour un même effort, accepter de percevoir un salaire plus faible que celui qu'ils auraient pu percevoir au sein du secteur privé, en raison de leur motivation intrinsèque à participer à la production de services générant des bénéfices sociaux ${ }^{6}$. Nous considérons ici que les administrations publiques, comme les organisations à but non lucratif, sont supposées

6. «Les bénéfices sociaux sont définis comme les externalités sociales et autres avantages dont profitent les parties extérieures à la transaction ou, plus spécifiquement, la société dans son ensemble » (PrESTON, 1989, p. 440, traduction de l'auteure). 
produire davantage de bénéfices sociaux que les entreprises privées profitables ${ }^{7}$ et qu'elles vont donc également attirer des travailleurs intrinsèquement motivés.

Cependant, approfondissant le modèle développé par A. E. PRESTON, de nouveaux modèles théoriques ont envisagé que les salariés motivés intrinsèquement par la production de biens ou services procurant des bénéfices sociaux pouvaient voir l'effort au travail tout à la fois comme une pénibilité et comme un investissement altruiste, engendrant ainsi et une peine et une satisfaction (voir par exemple BESLEY, GHATAK, 2005 ; DelfGaAuw, Dur, 2008 ; FrançOIS, 2007). Ainsi, par rapport aux modèles standard, qui représentent une fonction d'utilité ignorant la possibilité que les salariés soient intrinsèquement motivés (notée $U^{\prime}$ ), est inclus un troisième terme $\gamma_{\mathrm{e}}$ dans une fonction d'utilité comprenant déjà le salaire perçu et l'effort au travail. Ce troisième terme reflète le fait que l'agent retire une utilité positive de l'effort déployé à cette tâche à laquelle il accorde une valeur intrinsèque, c'est-à-dire que le coût marginal de l'effort est plus faible :

$$
\mathrm{U}^{\prime}=[\mathrm{w}(\mathrm{e}), \mathrm{e}] \rightarrow \mathrm{U}=\left[\mathrm{w}(\mathrm{e}), \mathrm{e}, \gamma_{\mathrm{e}}\right]
$$

La fonction d'utilité qu'un salarié $i$ retire de son emploi lorsqu'il participe à la production d'un bien générant des bénéfices sociaux peut être modélisée de la manière suivante, comme dans le modèle de Josse DELFGAAUw et Robert DuR (2008) :

$$
\mathrm{U}_{\mathrm{i}}=\mu\left(\mathrm{w}_{\mathrm{i}}\right)-\mathrm{c}\left(\mathrm{e}_{\mathrm{i}}\right)+\gamma_{\mathrm{i}} \mathrm{e}_{\mathrm{i}}
$$

où $\mu\left(\mathrm{w}_{\mathrm{i}}\right)$ représente l'utilité que le salarié $i$ retire du salaire $\mathrm{w}_{\mathrm{i}}$ perçu, $\mathrm{c}\left(\mathrm{e}_{\mathrm{i}}\right)$ est le coût associé à l'effort fourni et $\gamma_{i} \in[0, \bar{Y}]$ mesure le degré de motivation intrinsèque du salarié $i$; si $\gamma_{\mathrm{i}}=0$, le salarié n'est pas intrinsèquement motivé et sa fonction d'utilité est standard (elle correspond au modèle d'utilité classique U' $=[w(e), e]$ ). En d'autres termes, l'agent retire un bénéfice direct du fait de participer à une tâche qu'il considère comme utile à la société. Dans ce type de modélisation, l'action affecte directement l'agent en réduisant le coût d'exécution de la tâche ; ainsi, plus sa motivation intrinsèque est élevée, plus il est susceptible de retirer une utilité strictement positive du fait de fournir un effort.

Selon ce modèle, si l'on considère deux types de salariés $i$ et $j$ avec $\gamma_{\mathrm{i}}>\gamma_{\mathrm{j}}$, la motivation intrinsèque plus élevée du salarié $i$ peut se traduire de deux façons : soit, pour un même niveau d'effort $\left(\mathrm{e}_{\mathrm{i}}=\mathrm{e}_{\mathrm{j}}\right)$, le salarié $i$ est disposé à accepter un salaire plus faible que le salarié $j\left(\mathrm{w}_{\mathrm{i}}<\mathrm{w}_{\mathrm{j}}\right)$; soit, à salaire équivalent $\left(\mathrm{w}_{\mathrm{i}}=\mathrm{w}_{\mathrm{j}}\right)$, le salarié $i$ fournira un effort au travail plus élevé que le salarié $j\left(\mathrm{e}_{\mathrm{i}}>\mathrm{e}_{\mathrm{j}}\right)$. Par conséquent, un salarié sera davantage intrinsèquement motivé qu'un autre si l'effort plus élevé qu'il fournit (comparativement à cet autre salarié) n'est pas « compensé » par un niveau de salaire également plus élevé.

7. Du fait de leur intervention dans des secteurs d'activité tels que l'éducation, la santé ou l'action sociale et par la mise à disposition de biens et services qui profitent à tous les citoyens. 
Empiriquement, l'écart salarial observé entre les secteurs public et privé ne suffit donc pas à être interprété comme un don de travail. Parler de don n'est valide que dans la mesure où l'acceptation d'un salaire plus faible de la part des travailleurs du secteur public n'est pas compensée par un niveau d'effort également plus faible. De même, il n'est possible de parler de don de travail que si les employés du secteur public acceptent de fournir un effort supplémentaire par rapport à celui fourni par leurs homologues du secteur privé à but lucratif, toutes choses égales par ailleurs, c'est-à-dire à rémunérations et conditions de travail constantes.

Deux arguments plaident pour ne pas utiliser les comparaisons de salaire moyen entre secteurs public et privé comme preuves d'un éventuel don de travail. Tout d'abord, la composition des salaires dans la fonction publique est beaucoup plus standardisée que dans le secteur privé puisque les conditions de recrutement, de rémunération et de déroulement de carrière y sont déterminées par un statut fixé par décret correspondant au corps (dans la FPE ou la fonction publique hospitalière) ou au statut d'emploi (dans la fonction publique territoriale) d'appartenance de l'agent. Ensuite, la comparaison des salaires moyens entre secteurs public et privé cache de fortes disparités dans la distribution des rémunérations des deux secteurs ${ }^{8}$. De ce fait, il est préférable de fonder la mise en évidence d'un éventuel don de travail dans le secteur public sur une mesure de l'effort productif offert par les travailleurs. Nous utiliserons donc ici les heures supplémentaires non payées comme un indicateur de ce comportement prosocial - don de travail - de manière à observer les différences de comportement en termes d'effort de la part des salariés des secteurs public et privé, à caractéristiques individuelles et d'emploi (dont le salaire) identiques.

Seules deux études britanniques déjà citées suivent un programme similaire, en comparant les heures supplémentaires non rémunérées des salariés du secteur privé à but lucratif et du secteur qualifié de non profitable dans le BHPS, secteur qui regroupe les agents des administrations publiques, mais aussi les salariés des entreprises publiques et des associations à but non lucratif (VELIZIOTIS, 2010 ; GREGG et al., 2011). L'étude de P. GREGG et de ses co-auteurs (2011) montre que les salariés du secteur non lucratif ont une probabilité de faire ces heures non payées qui est supérieure de 12 points de pourcentage à celle des salariés du secteur privé à but lucratif. En outre, les auteurs mettent en évidence un élément de sélection volontaire dans le secteur non lucratif, car les salariés qui en sont issus et décident d'aller travailler dans le secteur privé à but lucratif sont ceux qui font significativement moins d'heures supplémentaires non payées. En revanche, dans l'étude de M. Veliziotis (2010), l'effet du don de travail n'est visible dans le secteur non lucratif que pour les salariés syndiqués ${ }^{9}$.

8. Claudio LuCIFORA et Dominique Meurs (2006) montrent qu'en France, en Grande-Bretagne et en Italie, les salariés du secteur public sont mieux rémunérés lorsqu'ils sont peu qualifiés et moins bien lorsqu'ils sont très qualifiés.

9. Certains auteurs ont montré que dans le secteur privé, lorsque les entreprises investissaient dans leur responsabilité sociale, elles pouvaient rémunérer leur main-d'œuvre à un salaire moyen plus faible (NYBORG, ZANG, 2013) et obtenaient un nombre d'heures supplémentaires non compensées plus élevé (LANFRANCHI, PEKOVIC, 2014). 


\section{Les autres explications théoriques des heures supplémentaires non compensées}

Les heures supplémentaires non compensées peuvent représenter tout autre chose qu'un don de travail. La littérature économique propose un grand nombre d'explications théoriques sur les raisons qui amènent les employés à « offrir » ces heures et qui doivent être, si possible, contrôlées dans l'analyse économétrique.

Parmi celles-ci, la théorie économique avance la méconnaissance par l'employeur du temps nécessaire à la réalisation d'une tâche, qui peut entraîner des heures supplémentaires non payées dans la mesure où le salarié dépasse le temps estimé pour l'exécution de l'activité considérée. Cet effet peut se révéler particulièrement important lorsque la tâche est complexe, notamment pour les cadres qui ont davantage de responsabilités et exercent des fonctions de gestion ou de supervision nécessitant des compétences multiples (BELL, HART, 1999). De plus, les cadres ne sont généralement pas soumis au même régime horaire, étant au forfait ou ayant des modalités plus flexibles de temps de travail (annualisation, horaires atypiques) que les autres catégories d'emploi, qui se calent sur des systèmes d'horaires plus rigides. Les cadres peuvent de ce fait se retrouver dans un environnement « où l'on ne compte pas ses heures » avec des évaluations fondées sur des objectifs à atteindre. Pour tenir compte de ces situations, nous retenons dans l'analyse économétrique des indicateurs de l'intensité du travail tels que l'imposition de délais pour la réalisation d'une tâche ou encore la mise en place d'objectifs de résultats. Ces indicateurs, comme le ressenti exprimé par le salarié de devoir toujours se presser, illustrent indirectement la difficulté à réaliser les tâches dans le temps de travail contractuel. Nous contrôlons également le nombre d'heures contractuel qu'un individu est censé travailler par semaine ainsi que le fait d'être à temps partiel, car ces deux indicateurs changent les conditions dans lesquelles chaque individu peut être considéré comme réalisant des heures supplémentaires impayées. Nous incluons également un indicateur identifiant les individus déclarant avoir des fonctions de gestion ou de supervision au travail. Enfin, comme David Bell et Robert HART (1999) montrent que les travailleurs non couverts par un syndicat sont plus susceptibles d'entreprendre des tâches n'ayant pas été préalablement négociées ou bornées en temps d'exécution, nous tiendrons compte, empiriquement, du fait que le salarié soit adhérent ou non d'un syndicat.

Une deuxième explication mentionnée par la littérature repose sur l'hypothèse selon laquelle les salariés déclarent réaliser ces heures non rémunérées dans le but de « communiquer » sur leur niveau d'effort et de motivation à leur employeur. Cet investissement en effort peut se justifier de façon offensive, dans l'espoir de percevoir une prime ou une promotion (ANGER, 2008 ; BRATTI, STAFFOLANI, 2007), mais aussi de façon plus défensive, pour protéger son emploi dans un environnement économique perçu comme incertain. Qu'il s'agisse d'une forme de compétition explicite de type tournoi, où le moins bon des travailleurs serait licencié, ou d'une menace implicite en cas de restructuration, certains travailleurs augmenteraient leur offre d'effort de manière à signaler leur bonne volonté relative et être maintenus dans l'emploi (PANNENBERG, 
2005). De ce fait, nous retenons dans notre analyse plusieurs variables concernant les préoccupations de carrière, telles que l'existence de critères d'évaluation du travail ayant des répercussions sur la rémunération ou les opportunités de carrière au sein de l'entreprise. De la même manière, un indicateur mesurant si les salaires perçus varient avec la performance est pris en compte ainsi qu'un indicateur de satisfaction relatif à la sécurité de l'emploi. Le niveau du salaire permet également de prendre en compte les préoccupations de carrière. En effet, si les heures supplémentaires non payées constituent un investissement pour augmenter ses revenus, sous la forme de paiements différés, l'effet du salaire doit être négatif : plus celui-ci est faible, plus le salarié donnera des heures en supplément dans l'espoir de le voir augmenter ${ }^{10}$. Cependant, un coefficient négatif associé à la variable de salaire peut aussi capturer un effet de coût d'opportunité : faire des heures supplémentaires gratuitement est plus coûteux lorsque le salaire est élevé. Suivant le même raisonnement, nous contrôlons le type de contrat de travail, les travailleurs employés à durée déterminée étant incités à fournir plus d'heures supplémentaires non compensées de façon à augmenter leur probabilité de passage à un contrat à durée indéterminée (ENGELLANDT, RIPHAHN, 2005 ; MEYER, WALLETTE, 2005).

L'organisation du travail choisie par l'employeur est une autre explication possible avancée par la littérature économique. Selon D. Bell et R. HART (1999), la performance productive d'une équipe serait fondée sur la capacité de ses membres à s'adapter au travail et à la personnalité des autres membres, en vue de maximiser la cohésion et la capacité productive collective. Ainsi, l'hétérogénéité de la productivité potentielle des membres d'une équipe est susceptible d'inciter les membres les moins productifs à devoir compenser leur moindre efficacité par une augmentation ponctuelle de leur temps de travail. De plus, en cas d'absence non programmée ou de licenciement inattendu d'un salarié, ses collègues directs peuvent fournir des heures gratuitement afin de maintenir la performance, et donc la réputation, de l'équipe et éventuellement son niveau de rémunération en cas de prime collective. Pour ces raisons, nous intégrons à notre modèle une variable mesurant la participation à un collectif de travail.

Un autre pan de la littérature souligne que la mise à disposition d'ordinateurs portables invite les travailleurs à empiéter sur leur temps privé, puisque l'entreprise leur fournit un moyen de le faire (HÜBLER, 2000). Les salariés n'ayant pas le temps de terminer les tâches prévues sur leur lieu de travail ont ainsi la possibilité de les achever à leur domicile, ce qui peut conduire à la déclaration d'heures supplémentaires non rémunérées. Empiriquement, nous contrôlons la possibilité d'augmenter la durée du travail non rémunérée grâce à ce travail nomade par une variable identifiant les salariés dotés d'un ordinateur portable. De surcroît, le volume des heures requises pour achever certaines tâches peut varier avec l'intensité du recours aux nouvelles pratiques organisationnelles et technologiques. En effet, les nouveaux modes d'organisation, qui s'appuient sur des outils organisationnels et informatiques innovants, requièrent de la

10. Le salaire actuel est ainsi susceptible d'être endogène, car il peut refléter des heures non payées travaillées dans le passé. 
part des salariés un apprentissage plus long et un niveau de formation plus important. Ce temps d'apprentissage est susceptible de déboucher sur la réalisation d'heures supplémentaires non payées. Ainsi, nous utilisons dans notre estimation des indicateurs continus de recours aux outils technologiques et managériaux, qui sont communs aux deux secteurs pour l'année $2006^{11}$.

Enfin, selon la théorie de l'échange de don (AKERLOF, 1982), certains salariés accepteraient de travailler sans être rémunérés de façon à récompenser l'employeur d'un traitement positif et équitable. Selon cette théorie, plus le salaire perçu par les travailleurs se rapproche de ce qu'ils considèrent comme étant « juste » à leur égard, plus ils fourniront d'efforts en retour. Ainsi, cet effet sera pris en compte empiriquement puisque nos données permettent de contrôler si le salarié considère que son travail est reconnu à sa juste valeur quand il fait le bilan de son apport à son entreprise et des bénéfices qu'il en retire. Par ailleurs, l'utilité ressentie dans un emploi dépend non seulement de la rémunération, mais de l'ensemble des conditions de travail offertes. De ce fait, l'argument de don - contre-don peut être étendu au-delà de l'équité salariale lorsque les travailleurs se sentent en général bien traités en termes de conditions de travail. Ainsi, certains auteurs, inspirés par les travaux de sociologie et de psychologie du travail, ont mis l'accent sur le fait que le sentiment d'être bien considéré serait hautement corrélé avec certaines caractéristiques organisationnelles telles que la faible influence de la hiérarchie, la latitude accordée pour la prise de décisions et l'autonomie (voir par exemple MinTZBerg, 1983 ; Frey, 1997). Cet ensemble de manifestations de confiance favoriserait la qualité de l'environnement au travail et la réciprocité de la part de l'employé, le poussant à rester plus longtemps sur son lieu de travail. Nous avons donc introduit des variables de contrôle telles qu' une bonne entente entre collègues ainsi que l'intérêt du travail mesuré par le fait d'apprendre régulièrement des choses nouvelles.

\section{Données}

La richesse du dispositif d'enquêtes couplées COI 2006-2007 sur les changements organisationnels et l'informatisation, qui inclut des établissements privés et publics, nous donne accès à un ensemble très large d'informations nécessaires pour neutraliser toutes les explications alternatives proposées par la littérature sur les heures supplémentaires non compensées. Nous pouvons ainsi véritablement évaluer la pertinence de l'hypothèse de don de travail.

La représentativité des salariés est définie à la fois par le champ de l'interrogation des employeurs (entreprises ou unités financièrement autonomes avec un critère de taille de 20 salariés et plus pour les entreprises, et de 10 salariés et plus pour les administrations) et par les contraintes liées à l'interrogation des salariés au sein de ces organisations : outre le mécanisme de tirage au sort des salariés dans l'organisation employeuse, les

11. Ces indicateurs ont été construits par BIGI et al. (2012) dans l'enquête COI. 
salariés échantillonnés ne sont interrogés qu'environ un an plus tard, ce qui implique qu'ils ont au moins un an d'ancienneté. Les analyses portent donc sur les salariés stables des entreprises et administrations enquêtées. Les fichiers d'étude représentent un peu plus de 14000 observations « salariées » dans près de 6400 entreprises privées et 955 observations « salariées » dans près de 300 administrations publiques.

Mais dans un souci de rendre plus homogènes et comparables nos deux populations d'intérêt (notamment au niveau des professions et des conditions de travail), et pour limiter l'influence des effets sectoriels, notre étude est réalisée sur un second champ, plus restreint, dans le secteur privé. L'absence de secteurs équivalents à l'industrie, à la construction et au commerce dans la FPE justifie que l'on ne prenne en compte, dans ce second champ, que le secteur des services aux entreprises ${ }^{12}$. Finalement, après traitement des observations manquantes ou incohérentes, notre échantillon d'étude contient 11731 salariés dans le secteur privé, dont 3398 dans le secteur privé restreint aux secteurs des services aux entreprises, et 951 salariés dans la FPE ${ }^{13}$.

L'enquête auprès des salariés inclut spécifiquement deux questions qui mesurent l'effort au travail : « Travaillez-vous au-delà de la durée habituelle ? » et « Avez-vous une compensation en salaire ou en repos en cas de dépassement des horaires de travail ? ». Le tableau 1 ci-dessous indique les moyennes des individus déclarant ainsi faire des heures supplémentaires, classés selon les différents secteurs (année 2006-2007). Ces premières statistiques descriptives montrent qu'a priori, il n'y a pas de différence forte de comportement en heures supplémentaires impayées entre la FPE et le secteur privé dans son ensemble. Toutefois, il semble que les salariés du secteur privé restreint aux services aux entreprises sont amenés à fournir plus

\section{TABLEAU 1 - Réalisation moyenne des heures de travail dans la FPE et dans les secteurs privé et privé restreint}

\begin{tabular}{lcccc}
\hline $\begin{array}{l}\text { Secteur } \\
\text { institutionnel }\end{array}$ & $\begin{array}{c}\text { Heures } \\
\text { hebdomadaires }\end{array}$ & $\begin{array}{c}\text { Déclaration d'heures } \\
\text { supplémentaires } \\
\text { (\% de travailleurs) }\end{array}$ & $\begin{array}{c}\text { Déclaration d'heures } \\
\text { supplémentaires non compensées } \\
\text { (\% travailleurs) }\end{array}$ & $\begin{array}{c}\text { Total } \\
\text { des } \\
\text { observations }\end{array}$ \\
\hline FPE & 37,2 & 72,8 & 22,3 & 951 \\
Privé & 38,1 & 72,5 & 24,0 & 11731 \\
Privé restreint & 37,1 & 72,0 & 26,4 & 3398 \\
\hline
\end{tabular}

Lecture : les agents de la FPE travaillent en moyenne 37,2 heures par semaine. 72,5 \% des salariés du privé dans son ensemble déclarent faire des heures supplémentaires (payées ou non) et $24 \%$ font des heures supplémentaires non compensées.

Champ : salariés stables (1 an d'ancienneté) des unités productives de 20 salariés et plus dans le secteur privé, de 10 salariés et plus dans le secteur public.

Sources : enquêtes COI 2006, COI-FP 2006, Dares, Institut national de la statistique et des études économiques (Insee), Direction générale de l'administration et de la fonction publique (DGAFP), Centre d'études de l'emploi (CEE).

12. Le secteur des services aux entreprises provenant de l'externalisation des fonctions auparavant intégrées au secteur industriel.

13. Nous rappelons que les agents de l'État considérés ici sont employés, hors périmètre du ministère de la Défense, dans des directions d'administration centrale ou déconcentrée, des services déconcentrés, des académies ou des établissements scolaires, et ne sont ni enseignants ni magistrats. 
d'heures supplémentaires non payées, puisqu'ils sont significativement plus nombreux à déclarer faire ces heures ${ }^{14}$.

Cependant, ce résultat ne peut être interprété tel quel. En effet, il est possible qu'il s'explique par des différences de caractéristiques spécifiques à chaque secteur, qui gommeraient la présence éventuelle d'un comportement prosocial.

Il existe par exemple un certain nombre de techniques de management de la main-d'œuvre et de modes d'organisation du travail qui sont essentiellement pratiqués dans le secteur privé et peuvent y influencer le comportement au travail des salariés. Comme le montre le tableau B des statistiques descriptives (annexe), les salariés du secteur privé font face à davantage de méthodes de management de type incitatif que leurs homologues du public. La variabilité des salaires, l'influence de l'évaluation du travail sur les possibilités d'augmentation ou encore le niveau de salaire sont significativement plus élevés. En revanche, les agents publics répondent plus fréquemment que l'évaluation de leur travail influence leurs opportunités de carrière. Les employés du secteur privé sont aussi davantage encadrés par l'imposition de délais ou d'objectifs de travail à réaliser. Par ailleurs, les deux groupes de travailleurs diffèrent également quant à leurs caractéristiques individuelles, les agents du public étant généralement plus âgés, diplômés et expérimentés ; le secteur public emploie plus de femmes que d'hommes, plus de travailleurs à temps partiel ou encore de travailleurs syndiqués que le secteur privé. Ces différences peuvent participer à expliquer l'écart de comportement observé entre ces deux secteurs en termes d'heures supplémentaires non payées.

De manière à dépasser cet estimateur naïf de la différence de probabilité de faire ces heures entre les deux secteurs, nous devons analyser ce comportement en contrôlant au mieux les effets provenant des variables dont la théorie économique prédit qu'elles peuvent influencer l'effort et qui ont été discutées dans la section précédente. De cette façon, nous pourrons éventuellement isoler les effets provenant résiduellement d'une possible motivation prosociale. Dans notre analyse économétrique, nous conservons aussi un certain nombre de variables individuelles, comme le genre, l'âge, le niveau d'études, la catégorie socioprofessionnelle et le nombre d'années d'ancienneté dans l'entreprise. Et nous incluons une variable de contrôle pour le nombre de personnes à charge au sein du ménage, car cette situation est susceptible d'affecter le coût d'opportunité de faire des heures supplémentaires non payées au regard de l'impératif de conciliation entre vie professionnelle et vie familiale. Concernant la sélection des variables caractérisant l'emploi, le dispositif $C O I$ interroge assez largement les salariés sur leurs conditions de travail et sur leur ressenti dans l'emploi, nous permettant ainsi de tenir compte des heures non rémunérées qui proviennent non pas d'un don de travail, mais plutôt d'une exigence du poste ${ }^{15}$.

14. Le test de comparaison de moyenne de déclaration des heures supplémentaires non payées entre les salariés du secteur privé restreint et de la FPE affiche une différence significative à $1 \%$.

15. L'ensemble des variables explicatives utilisées est détaillé dans le tableau A de l'annexe statistique. 


\section{Méthodologie économétrique}

Nous proposons d'analyser en trois étapes la probabilité de faire des heures supplémentaires non payées dans le secteur privé et la FPE. Dans un premier temps, à l'aide d'une régression biprobit, nous mettons en évidence l'effet d'appartenir au secteur public sur la probabilité de faire ces heures, en tenant compte de l'éventuelle sélection volontaire dont résulte l'appartenance au secteur public. Nous proposons ensuite de faire ressortir les déterminants de ces heures « gratuites » de façon spécifique dans chacun des secteurs à l'aide de régressions probit séparées conduites en leur sein. Enfin, dans une troisième étape, nous évaluons les déterminants qui pèsent le plus sur la différence d'heures supplémentaires non payées entre le secteur privé et la FPE au moyen de la méthode de décomposition détaillée de Robert FAIRLIE (2005).

\section{Estimation de l'effet de l'appartenance au secteur public sur la probabilité de faire des heures supplémentaires non compensées}

Selon l'hypothèse théorique à étudier, les agents du secteur public, motivés par la participation à la production de bénéfices sociaux, sont prêts à faire un don d'effort, compte tenu des méthodes d'incitation, du salaire, des modes de définition des tâches, des conditions de travail, etc. Nous cherchons donc à estimer l'effet de l'appartenance au secteur public sur la probabilité de faire des heures supplémentaires non payées, toutes choses égales par ailleurs, à l'aide d'un modèle probit bivarié. Le choix d'un secteur d'activité plutôt qu'un autre doit être pris en compte, car il peut être lié aux sources de motivation des individus et influer sur leur comportement ultérieur d'offre d'effort.

Pour tenir compte des variables inobservables qui expliqueraient à la fois la sélection volontaire dans la FPE et l'offre d'heures supplémentaires de travail, nous estimons donc simultanément les facteurs qui expliquent ces deux décisions.

Nous notons $Y_{1}$, la variable observée dichotomique prenant la valeur 1 si l'individu travaille dans le secteur public et 0 sinon, tandis que la variable $Y_{2}$, dichotomique également, est égale à 1 si l'individu $i$ déclare travailler des heures supplémentaires non payées et 0 sinon. Elles sont donc définies de la manière suivante :

$$
Y_{1}=1 \text { si } Y_{1}^{*}>0, Y_{1}=0 \text { sinon }
$$

et

$$
Y_{2}=1 \text { si } Y_{2}^{*}>0, Y_{2}=0 \text { sinon }
$$

où $Y_{1}^{*}$ et $Y_{2}^{*}$ sont des variables latentes mesurant respectivement les bénéfices inobservables associés à un emploi dans la FPE et ceux liés au fait d'effectuer des heures supplémentaires non payées. Nous considérons le modèle probit bivarié suivant :

$$
\left\{\begin{array}{l}
Y_{1}^{*}=X_{1} \beta_{10}+W_{1} \beta_{11}+Z_{1} \beta_{12}+\varepsilon_{1} \\
Y_{2}^{*}=X_{1} \beta_{20}+W_{1} \beta_{21}+Z_{1} \beta_{22}+\varepsilon_{2}
\end{array}\right.
$$


Afin d'estimer convenablement l'effet de l'appartenance à la FPE sur la probabilité d'effectuer ces heures, notre modèle contient deux ensembles de variables exogènes : les caractéristiques individuelles rassemblées dans le vecteur $\mathrm{X}_{1}$, telles que l'âge, le genre ou la catégorie socioprofessionnelle, et les caractéristiques de l'emploi dans le vecteur $\mathrm{W}_{1}$, discutées plus haut. Pour des raisons liées aux problèmes d'identification, ces deux vecteurs sont les mêmes dans les deux équations.

Aussi, il est possible que les agents de la FPE n'aient pas choisi leur secteur en raison de leur préférence unique pour les missions de service public rendues, mais pour pouvoir bénéficier de conditions de travail plus agréables ou encore en raison d'un comportement d'aversion pour le risque puisque le statut de fonctionnaire en France est extrêmement protecteur en termes de sécurité de l'emploi, de choix de mobilité géographique, de prévisibilité des rémunérations et de carrière. Toutefois, en contrôlant différentes variables caractérisant les conditions de travail et d'organisation de l'emploi dans l'équation du choix de secteur, nous pouvons neutraliser cette éventualité. En outre, si effectivement l'aversion pour le risque de perdre son emploi peut expliquer le choix du secteur public, cela ne justifie sans doute pas pour autant le fait de faire ces heures impayées au sein de ce secteur.

Les instruments nécessaires à l'identification du modèle probit bivarié sont inclus dans le vecteur $\mathrm{Z}_{1}$ de la première équation du modèle ${ }^{16}(1)$.

Le premier instrument envisagé consiste en un indicateur de la profession d'enseignant exercée par le père ou la mère du salarié échantillonné, le second identifie si la nationalité de l'un ou l'autre des parents est française. Ainsi, selon une hypothèse de reproduction sociale, le choix du secteur public par un individu est souvent influencé par l'appartenance d'au moins un de ses parents au secteur public ou à un secteur fournissant des services non marchands à la société ${ }^{17}$ (AUDIER, 2000 ; Bigi et al., 2012). Par ailleurs, nous faisons l'hypothèse que la nationalité française d'un des deux parents peut prédisposer un individu à rejoindre le secteur public pour des raisons d'accès, de culture ou de maîtrise de la langue ${ }^{18}$. En effet, pour un certain nombre d'emplois dans l'administration française, il est obligatoire de détenir la nationalité française, et l'origine étrangère implique un coût lié à la naturalisation pour l'entrée dans l'administration française ${ }^{19}$.

16. La présence explicite d'instruments n'est pas strictement nécessaire pour réaliser l'estimation de ce modèle. Toutefois, une analyse de Monte-Carlo a permis de montrer l'importance des relations d'exclusion pour améliorer la qualité de l'estimation des paramètres (MONFARDINI, RADICE, 2008).

17. Cet instrument peut être discuté dans la mesure où il peut potentiellement influencer la motivation prosociale des enfants et, dans ce cas, avoir un effet direct sur les heures supplémentaires non payées. Compte tenu de cette critique, nous avons réalisé les estimations en considérant différentes combinaisons possibles des instruments proposés.

18. Le rapport de Yannick L'HORTY (2016) sur les discriminations dans l'accès à l'emploi public rapporte l'influence négative du fait d'être né hors de France métropolitaine sur la réussite aux épreuves écrites comme orales des concours d'entrée dans la fonction publique.

19. Signalons toutefois que depuis 2005, l'appartenance à un pays de l'Union européenne ou à un autre État participant à l'accord sur l'Espace économique européen (EEE), ou à la Suisse, est une condition considérée comme absolument identique à la nationalité française pour l'accès à la fonction publique. 
Les résidus des deux équations $\varepsilon_{1}$ et $\varepsilon_{2}$ sont supposés suivre une loi normale bivariée d'espérances nulles et de matrice variance-covariance $\Sigma$ :

$$
\Sigma=\left(\begin{array}{ll}
1 & \rho_{12} \\
\rho_{12} & 1
\end{array}\right)
$$

les éléments diagonaux étant normalisés à l'unité, tandis que $\rho_{12}$ représente le coefficient de corrélation entre les deux termes d'erreur ${ }^{20}$.

\section{Analyse des déterminants des heures supplémentaires non compensées spécifiques à chaque secteur}

De manière à identifier les spécificités propres à chaque secteur dans les déterminants des heures supplémentaires non payées, nous estimons à l'aide d'un modèle probit deux équations séparément, qui expliquent la probabilité de faire cet effort gratuit.

Le résultat est déterminé par une variable latente $Y_{i}^{*}$ pub , positive si le travailleur du public déclare faire des heures supplémentaires non payées (dans ce cas, $Y_{i}^{p u b}=1$ ) et négative sinon (auquel cas, $Y_{i}^{p u b}=0$ ). De même, $Y_{i}^{*}$ priv est une variable latente positive si le travailleur du secteur privé déclare faire ces heures $\left(Y_{i}^{p r i v}=1\right)$ et négative sinon $\left(Y_{i}^{\text {priv }}=0\right)$.

La propension individuelle à faire ces heures est ainsi définie dans chacun des secteurs :

$$
\begin{gathered}
Y_{i}^{\text {pub }}=F\left(X_{i}^{p u b} \alpha^{p u b}+W_{i}^{\text {pub }} \beta^{\text {pub }}\right) \\
Y_{i}^{\text {priv }}=F\left(X_{i}^{\text {priv }} \alpha^{\text {priv }}+W_{i}^{\text {privb }} \beta^{\text {priv }}\right),
\end{gathered}
$$

où les vecteurs $\mathrm{X}_{\mathrm{i}}$ et $\mathrm{W}_{\mathrm{i}}$ contiennent les mêmes caractéristiques individuelles et de l'emploi que dans le modèle (1) estimé préalablement.

Une exception toutefois parmi les variables pouvant expliquer le choix de faire ces heures : le sentiment de peur de certains travailleurs de perdre leur emploi. Cette variable pouvant influencer l'effort dans le privé pour des raisons incitatives a également la particularité de ne pas être considérée comme vraisemblable par les agents publics ayant répondu à l'enquête COI. Ainsi, un seul agent de la FPE a déclaré craindre pour son emploi, contre plus de $10 \%$ des salariés échantillonnés dans le secteur privé. Nous n'incluons donc la variable relative à la peur de perdre son emploi que dans l'équation du secteur privé.

20. Un test de Wald permet de vérifier directement l'hypothèse d'endogénéité de la sélection dans le secteur public sur le comportement d'offre d'heures supplémentaires. Si les heures supplémentaires impayées dans la FPE sont effectivement expliquées par des variables inobservables ayant également influencé le choix de ce secteur, nous nous attendons à ce que le coefficient de corrélation entre les termes d'erreur $\varepsilon_{1}$ et $\varepsilon_{2}$ soit positif. 


\section{Décomposition détaillée de l'écart de probabilité de faire des heures supplémentaires non compensées entre les deux secteurs}

Le principe de la méthode de décomposition détaillée de R. FAIRLIE (2005) consiste à décomposer l'écart observé de probabilité de faire des heures supplémentaires non payées entre les secteurs public et privé. Cette méthode de décomposition propose une analyse équivalente à celle initialement proposée par Alan BLINDER et Ronald OAXACA en $1973^{21}$, mais pour des modèles non linéaires avec une variable d'intérêt binaire, comme c'est le cas pour notre variable mesurant l'existence d'heures supplémentaires non compensées.

Il s'agit alors de décomposer la différence de probabilités moyennes de faire ces heures en deux parties, entre le secteur privé et la FPE. La première partie de la décomposition fait référence à une différence attribuable à la distribution des caractéristiques observables (la partie expliquée) et la deuxième représente une différence attribuable aux effets de ces caractéristiques, ou encore au processus de détermination de ces caractéristiques (soit la composante inexpliquée). Cette partie capte aussi la part due aux caractéristiques inobservables ${ }^{22}$.

$$
\begin{aligned}
\bar{Y}^{\text {priv }}-\bar{Y}^{\text {pub }} & =\left[\sum_{i=1}^{N^{\text {priv }}} \frac{F\left(X_{i}^{\text {priv }} \cdot \hat{\alpha}^{\text {priv }}+W_{i}^{\text {priv }} \cdot \hat{\beta}^{\text {priv }}\right)}{N^{\text {priv }}}\right. \\
& \left.-\sum_{i=1}^{N \text { pub }} \frac{F\left(X_{i}^{\text {pub }} \cdot \hat{\alpha}^{\text {priv }}+W_{i}^{\text {pub }} \cdot \hat{\beta}^{\text {priv }}\right)}{N^{\text {pub }}}\right] \\
& +\left[\sum_{i=1}^{N^{\text {pub }}} \frac{F\left(X_{i}^{\text {pub }} \cdot \hat{\alpha}^{\text {priv }}+W_{i}^{\text {pub }} \cdot \hat{\beta}^{\text {priv }}\right)}{N^{\text {pub }}}-\sum_{i=1}^{N^{p u b}} \frac{F\left(X_{i}^{\text {pub }} \cdot \hat{\alpha}^{\text {pub }}+W_{i}^{\text {pub }} \cdot \hat{\beta}^{\text {pub }}\right)}{N^{\text {pub }}}\right]
\end{aligned}
$$

$\overline{Y^{\jmath}}$ est la probabilité moyenne de déclarer faire des heures supplémentaires impayées dans la population $j(\mathrm{j}=$ priv, pub), soit pour les salariés du privé et du public respectivement. $X^{j}$ et $W^{j}$ correspondent à la distribution des caractéristiques observables, individuelles et de l'emploi respectivement, au sein de la population considérée. $\widehat{\alpha^{J}}$ et $\widehat{\beta^{J}}$ sont les coefficients estimés, affectés aux caractéristiques individuelles et d'emploi. $N^{j}$ fait référence à la taille de l'échantillon de chaque sous population et $\mathrm{F}($.) est la fonction de distribution cumulative qui suit une loi normale.

La littérature propose différentes méthodes de pondération fournissant des résultats d'estimation différents. Nous choisissons ici d'utiliser les salariés du secteur

\footnotetext{
21. A. Blinder (1973) et R. OAXACA (1973) proposent une décomposition linéaire pour expliquer les discriminations existantes sur le marché du travail entre deux groupes de population.

22. R. FAIRLIE (2005) ne s'intéresse de près qu'à la partie expliquée en raison de la difficulté d'interpréter le résultat de la partie inexpliquée (problème d'identification lié au fait que le résultat dépend de la modalité mise en référence pour la variable explicative).
} 
privé comme population de référence, puisque les coefficients estimés de la régression probit des heures supplémentaires non payées pour les salariés du privé sont utilisés pour pondérer le premier terme de l'expression (la partie expliquée), alors que la distribution des caractéristiques des agents du public est mobilisée pour pondérer le second terme de l'expression. On suppose en effet que dans le secteur privé ces heures sont fonction des caractéristiques observables, tandis que ce comportement serait potentiellement plus fréquent chez les agents du secteur public, en raison de données inobservables liées à leur motivation prosociale.

Afin d'estimer la décomposition de R. FAIRLIE, il est nécessaire de disposer d'échantillons de même taille. Or, notre échantillon de salariés du privé est très supérieur en nombre à celui de la FPE. Nous devons donc apparier un nombre de salariés du privé égal au nombre d'agents publics et faire la décomposition proposée. Pour ce faire, notre méthode retient trois étapes :

1) estimer pour les deux groupes de salariés la probabilité de faire des heures supplémentaires non compensées, puis prévoir pour chaque individu cette probabilité au moyen du modèle estimé ;

2) tirer de façon aléatoire dans l'échantillon des salariés du privé un sous-échantillon dont l'effectif est égal au nombre d'agents de la FPE. Les individus des deux échantillons de taille comparable sont ensuite ordonnés selon la valeur prévue de la probabilité estimée en première étape et appariés selon leur rang ;

3) la décomposition est finalement réalisée sur l'échantillon apparié.

Suivant les enseignements de R. FAIRLIE, nous avons réalisé 500 réplications de cette procédure, et les contributions interprétées des différentes variables explicatives de la décomposition sont les moyennes de leurs contributions dans ces 500 réplications.

Signalons cependant dès à présent la prudence requise dans l'interprétation des résultats des méthodes de décomposition. Ces méthodes présentent de fait deux principales limites relatives à une possible « dépendance de sentier » et à des problèmes d'« identification ». La première limite renvoie au fait que l'ordre d'introduction des variables influence les résultats en raison de la non-linéarité de l'équation de décomposition. En effet, dans un modèle de régression de variable qualitative, l'hypothèse d'additivité des différentes contributions n'est pas vérifiée. La solution proposée par R. FAIRLIE est de rendre aléatoire l'ordre d'entrée des variables dans chacune des 500 réplications à l'aide d'une option spécifique lors du calcul de la décomposition, puis de faire la moyenne des contributions de chaque variable dans chacun des ordres dans chacune des compositions possibles ${ }^{23}$. La deuxième limite est que les résultats obtenus dépendent de la modalité de référence choisie dans le cas de variables explicatives qualitatives (NARCY, 2013b).

23. La procédure d'estimation de la contribution relative de chaque variable consiste en un certain nombre d'étapes techniques qui sont présentées en détail par R. FAIRLIE dans son article de référence (2005). 


\section{Résultats}

\section{Résultats du modèle probit bivarié}

Les résultats de l'estimation de l'équation de sélection sont présentés dans les colonnes 4 et 5 du tableau 2 , tandis que les résultats de l'estimation des heures supplémentaires impayées (colonnes 2 et 3 du tableau 2) montrent qu'il n'y a pas de différence vraisemblable de probabilité de faire ces heures entre les deux secteurs ${ }^{24}$. De plus, le coefficient de corrélation entre les termes d'erreur $\varepsilon_{1}$ et $\varepsilon_{2}$ est négatif et non significatif, ce qui signifie que les heures supplémentaires non payées dans la FPE ne sont pas expliquées par des variables inobservables ayant influencé le choix du secteur ${ }^{25}$.

En outre, l'effet de l'appartenance à la FPE sur la probabilité de faire ces heures impayées demeure non significatif lorsque l'analyse est circonscrite à un secteur privé restreint, constitué du seul secteur des services aux entreprises, de façon à le rendre le plus comparable possible aux activités exercées dans la FPE (colonnes 6 et 7 du tableau 2).

TABLEAU 2 - Estimation de modèles probit bivarié avec effet de sélection (considérant le secteur privé dans son ensemble et le secteur privé restreint aux services aux entreprises)



Notes : ${ }^{+}$Heures supplémentaires non compensées.

${ }^{++}$L'effet des caractéristiques individuelles et d'emploi contrôlées dans l'équation n'est pas présenté ici, car seuls nous intéressent l'effet de l'appartenance à la FPE sur ces heures et la corrélation entre les termes d'erreur (tableau de résultat complet disponible auprès de l'auteure).

Significativité à : *** $1 \%, * * 5 \%, * 10 \%$.

Champ : salariés stables (1 an d'ancienneté) des unités productives de 20 salariés et plus dans le secteur privé, de 10 salariés et plus dans le secteur public.

Sources : enquête COI 2006 (Insee, Dares) ; COI-FP 2006 (Dares, DGAFP, CEE).

24. Ce résultat est resté stable quelles que soient les combinaisons d'instruments testées : le coefficient affecté à la variable d'appartenance au secteur public est toujours positif, mais non significatif.

25. En excluant les variables caractérisant l'emploi occupé dans l'équation du choix de secteur, les résultats restent inchangés. 
De même, le coefficient de corrélation $\rho$ est non significatif, confirmant que les raisons qui justifient le choix du secteur public sont sans effet sur la probabilité de faire ces heures gratuites ${ }^{26}$. Cela dit, si notre hypothèse sur l'existence d'un don de travail se traduisant par des heures supplémentaires non compensées plus probable chez les agents de la FPE est invalidée, cela ne signifie pas que ces derniers ne sont pas dotés d'une motivation prosociale plus élevée. Il se pourrait en effet que ce don passe par un canal d'effort différent.

\section{Résultats du modèle probit estimé séparément pour chaque secteur}

L'estimation de l'offre d'heures supplémentaires par secteur (tableau 3) permet de vérifier si les variables expliquant ce choix de la part des salariés diffèrent selon leur secteur d'appartenance, et révèle ainsi que certains choix des employeurs en termes d'incitation et d'organisation du travail y sont plus ou moins motivants.

Nous estimons séparément la probabilité de faire des heures supplémentaires non payées dans le secteur privé dans son ensemble, dans le secteur privé restreint aux services aux entreprises et dans la FPE, à l'aide d'un modèle probit et sans tenir compte de l'effet de sélection, car nous considérons que le biais d'endogénéité n'est pas important au vu des résultats obtenus précédemment. Les coefficients estimés associés aux caractéristiques des salariés du secteur privé et privé restreint, déterminant leur comportement en termes d'heures supplémentaires non compensées, sont présentés respectivement dans les colonnes 2 et 3 du tableau, tandis que la colonne 4 reporte les coefficients estimés des déterminants de ces heures pour les agents de la FPE.

TABLEAU 3 - Estimation des heures supplémentaires non compensées dans le secteur privé dans son ensemble, dans le secteur privé restreint et dans la FPE (probit)

\begin{tabular}{lcc|cc|cc}
\hline & \multicolumn{2}{c|}{ Privé } & \multicolumn{2}{c|}{ Privé restreint } & \multicolumn{2}{c}{ FPE } \\
\cline { 2 - 7 } & Coefficient & $\sigma$ & Coefficient & $\sigma$ & Coefficient & $\sigma$ \\
\hline Homme & $-0,018$ & 0,033 & $-0,076$ & 0,0601 & $-0,029$ & 0,115 \\
Personnes à charge & $-0,026^{* *}$ & 0,012 & $-0,035$ & 0,0259 & $-0,045$ & 0,050 \\
Âge & $0,003^{*}$ & 0,001 & $-0,027$ & 0,0229 & $0,016^{* *}$ & 0,007 \\
Âge au carré & 0,000 & 0,000 & 0,000 & 0,0002 & 0,000 & 0,000 \\
\hline Diplôme (réf : primaire) & & & & & & \\
Technique & $-0,028$ & 0,058 & 0,001 & 0,1238 & 0,172 & 0,226 \\
Baccalauréat & 0,004 & 0,064 & $-0,041$ & 0,1347 & $0,403^{*}$ & 0,223 \\
Supérieur court & $0,214^{* * * *}$ & 0,065 & 0,119 & 0,1354 & $0,502^{* *}$ & 0,233 \\
Supérieur long & $0,358^{* * *}$ & 0,070 & $0,344^{* *}$ & 0,1387 & $0,427^{*}$ & 0,249 \\
\hline
\end{tabular}

26. Des analyses ont également été menées en excluant les emplois précaires de l'échantillon d'étude (intérim, saisonniers, stage, etc.) et en ne considérant que les salariés en contrat à durée indéterminée (CDI). Les résultats révèlent très peu de changements. 


\begin{tabular}{|c|c|c|c|c|c|c|}
\hline & \multicolumn{2}{|c|}{ Privé } & \multicolumn{2}{|c|}{ Privé restreint } & \multicolumn{2}{|c|}{ FPE } \\
\hline & Coefficient & $\sigma$ & Coefficient & $\sigma$ & Coefficient & $\sigma$ \\
\hline \multicolumn{7}{|l|}{ Ancienneté (réf : 5-10 ans) } \\
\hline $1-3$ ans & 0,073 & 0,053 & $-0,036$ & 0,0949 & $0,918^{* * * *}$ & 0,313 \\
\hline 3-5 ans & $0,126^{* *}$ & 0,049 & $0,155^{*}$ & 0,0858 & $0,584^{* *}$ & 0,240 \\
\hline$>10$ ans & $-0,017$ & 0,038 & 0,011 & 0,0701 & $0,415^{* *}$ & 0,173 \\
\hline \multicolumn{7}{|c|}{$\begin{array}{l}\text { Profession et catégorie socioprofessionnelle (PCS) } \\
\text { (réf : profession intermédiaire) }\end{array}$} \\
\hline Cadre & $0,310^{* * * *}$ & 0,044 & $0,383^{* * *}$ & 0,0769 & $0,652^{* * * *}$ & 0,188 \\
\hline Employé & $-0,238^{* * * *}$ & 0,045 & $-0,366^{* * *}$ & 0,0870 & $-0,459^{* * *}$ & 0,139 \\
\hline Ouvrier & $-0,749^{* * * *}$ & 0,045 & $-0,817^{* * *}$ & 0,0958 & $-0,532^{* *}$ & 0,260 \\
\hline \multicolumn{7}{|l|}{ Rapport au temps } \\
\hline Délai & $-0,068^{* *}$ & 0,029 & $-0,013$ & 0,053 & $-0,008$ & 0,108 \\
\hline Objectif & $0,087^{* * *}$ & 0,031 & 0,068 & 0,057 & $-0,014$ & 0,111 \\
\hline Stress & $0,171^{* * * *}$ & 0,028 & $0,179^{* * * *}$ & 0,052 & $0,239^{* *}$ & 0,239 \\
\hline Heures hebdomadaires & $0,022^{* * * *}$ & 0,002 & $0,018^{* * * *}$ & 0,004 & $0,023^{* *}$ & 0,011 \\
\hline Temps partiel & $0,184^{* * * *}$ & 0,064 & $0,324^{* * *}$ & 0,107 & $0,421^{* * *}$ & 0,177 \\
\hline Supervision & $-0,017$ & 0,032 & $-0,036$ & 0,062 & 0,064 & 0,125 \\
\hline Contrôle & $-0,020$ & 0,031 & $-0,062$ & 0,057 & 0,031 & 0,120 \\
\hline Syndicat & $-0,081^{*}$ & 0,041 & $-0,216^{* * * *}$ & 0,079 & $-0,182^{*}$ & 0,109 \\
\hline \multicolumn{7}{|l|}{ Effet de signal } \\
\hline Opportunité de carrière & $-0,041$ & 0,037 & $-0,047$ & 0,066 & 0,020 & 0,119 \\
\hline Emploi risqué & $0,160^{* * *}$ & 0,046 & $0,198^{* *}$ & 0,084 & - & - \\
\hline Augmentation de salaire & $-0,016$ & 0,037 & $-0,001$ & 0,067 & 0,167 & 0,141 \\
\hline Salaire variable & 0,005 & 0,030 & 0,013 & 0,056 & $-0,313^{* *}$ & 0,134 \\
\hline Log salaire par quinzaine & 0,048 & 0,038 & $-0,016$ & 0,069 & $-0,178$ & 0,217 \\
\hline \multicolumn{7}{|l|}{ Autre organisation du travail } \\
\hline Groupe & $0,103^{* * *}$ & 0,035 & $0,154^{* *}$ & 0,078 & $-0,059$ & 0,127 \\
\hline IU management & $-0,191^{* * * *}$ & 0,031 & $-0,022$ & 0,056 & $-0,182^{*}$ & 0,112 \\
\hline $\begin{array}{l}\text { IU technologies de l'information et de la } \\
\text { communication (TIC) }\end{array}$ & $0,063^{*}$ & 0,032 & $-0,008$ & 0,059 & 0,103 & 0,146 \\
\hline Ordinateur portable & $0,147^{* * * *}$ & 0,036 & $0,154^{* *}$ & 0,063 & $0,305^{* *}$ & 0,125 \\
\hline \multicolumn{7}{|l|}{ Don - contre-don } \\
\hline Ambiance entre collègues & $-0,009$ & 0,036 & 0,025 & 0,068 & $-0,057$ & 0,131 \\
\hline Nouvelle & 0,053 & 0,037 & 0,102 & 0,073 & 0,112 & 0,154 \\
\hline Reconnaissance & $-0,255^{* * *}$ & 0,030 & $-0,228^{* * * *}$ & 0,055 & $-0,167$ & 0,108 \\
\hline Zone d'habitation : Paris & $0,074^{* *}$ & 0,037 & $0,115^{*}$ & 0,062 & 0,084 & 0,143 \\
\hline Constante & $-2,062^{* * *}$ & 0,258 & $-0,790$ & 0,632 & $-2,020$ & 1,283 \\
\hline $\mathbf{N}$ & \multicolumn{2}{|c|}{11639} & \multicolumn{2}{|c|}{3383} & \multicolumn{2}{|c|}{949} \\
\hline
\end{tabular}

Significativité à : *** $1 \%, * * 5 \%, * 10 \%$.

Champ : salariés stables (1 an d'ancienneté) des unités productives de 20 salariés et plus dans le secteur privé, de 10 salariés et plus dans le secteur public.

Sources : enquête COI 2006 (Insee-Dares) ; COI-FP 2006 (Dares, DGAFP, CEE).

Les résultats de cette analyse montrent tout d'abord que les heures supplémentaires non rémunérées sont principalement liées à des contraintes de type organisationnel. Confirmant certaines des explications formulées par D. BELL et R. HART (1999) sur l'incertitude du temps nécessaire à la réalisation d'une tâche, nous trouvons que la probabilité de faire ces heures est significativement plus élevée pour les cadres et les 
plus diplômés. De même, le nombre d'heures de travail hebdomadaires augmente la probabilité de faire ces heures. Ce ne serait donc pas parce que les employeurs ont allongé la durée du temps de travail hebdomadaire en fonction de ce qu'ils croient être la difficulté de la tâche que certains ne se sentent pas obligés de fournir des heures de travail au-delà de celles prévues par le contrat. Le sentiment d'être débordé, variable traduisant une pression temporelle et éventuellement un sentiment d'urgence dans l'emploi, influe aussi positivement sur ces heures.

Les résultats montrent par ailleurs que l'emploi de certaines méthodes d'organisation du travail et de gestion des ressources humaines, efficaces au sein du secteur privé, n'a pas le même effet sur le fait de fournir un effort supplémentaire chez les agents de la FPE. Ainsi, conformément aux théories traditionnelles sur les incitations au travail, la variable indicatrice de la peur de perdre son emploi constitue une menace visiblement pertinente pour influencer ce comportement d'heures supplémentaires non payées dans le secteur privé, mais est sans objet dans la $\mathrm{FPE}^{27}$.

Enfin, nous trouvons que le recours à certaines pratiques supposément incitatives contribue à réduire le comportement de sur-effort parmi les agents de la FPE. Par exemple, le fait d'avoir une partie ou la totalité de son salaire variable réduit significativement la probabilité de déclarer faire des heures supplémentaires non payées chez les agents de la FPE, alors que cette variabilité du salaire a un effet positif sur la tendance à exécuter ces heures dans les deux échantillons du privé considérés (l'effet est cependant non significatif). On peut interpréter ce résultat comme une mise en évidence indirecte de la motivation intrinsèque des agents. La théorie de l'éviction de la motivation intrinsèque (FREY, 1997) suggère ainsi que la motivation des travailleurs du secteur public et associatif ne devrait pas être entretenue par les mêmes moyens, mis en œuvre par le management des ressources humaines, que ceux généralement pratiqués dans le secteur privé. Dans un contexte de mise en place de la culture du résultat, la rémunération des agents publics ne se limite plus au seul traitement indiciaire, mais est désormais complétée par diverses primes liées à la performance. Ces éléments incitatifs, se substituant en quelque sorte à la volonté propre de l'individu de fournir l'effort nécessaire à l' accomplissement de la tâche, peuvent créer un sentiment de rejet chez les agents, évinçant alors la motivation intrinsèque initialement présente (FrEY, 1997 ; BÉNABOU, Tirole, 2003). D'autres résultats de la littérature empirique ont d'ailleurs montré que les changements managériaux ont conduit les travailleurs dans le secteur public non seulement à se déclarer moins impliqués que leurs homologues du privé, mais aussi à se sentir moins bien traités qu'auparavant (BIGI et al., 2012).

En outre, les résultats au sein de la FPE témoignent d'une corrélation négative entre le comportement d'heures supplémentaires impayées et le nombre d'années passées dans l'organisation publique, tandis que cet effet ne s'observe pas dans le

27. Pour veiller à la comparabilité, en termes de caractéristiques, de tâches et de contrats, entre les répondants des deux secteurs, nous estimons une nouvelle fois le probit bivarié en restreignant notre échantillon aux seuls individus déclarant ne pas craindre pour leur emploi : les résultats restent quasiment inchangés. 
secteur privé. Le plafonnement des possibilités d'avancement de carrière ou de rémunération des fonctionnaires, fortement lié à l'ancienneté (additionné à une sécurité de l'emploi maintenue), peut en effet avoir pour conséquence une baisse de la motivation pour l'effort que l'agent développe parfois en début de carrière. Cependant, ce résultat pourrait tout aussi bien être mis en lien avec l'existence potentielle d'une motivation intrinsèque plus élevée des agents du secteur public, qui s'érode au fil du temps passé dans l'organisation. Plusieurs autres résultats de la littérature empirique ont mis en évidence que la longueur du temps passé dans les organisations publiques peut affecter la motivation de service public (MOYNIHAN, PANDEY, 2007 ; BUURMAn et al., 2012). Ainsi, Donald Moynihan et Sanjay PANDEY (2007) postulent qu'en raison de la nature des objectifs poursuivis et de l'importance accordée aux éléments intrinsèques de l'emploi, en particulier dans les secteurs des soins et des services sociaux, les salariés du secteur public développeraient de la frustration et du découragement au fil du temps s'ils perçoivent que les institutions les limitent dans l'accomplissement de leur mission. Aussi, les lourdeurs bureaucratiques et organisationnelles telles que l'excès de règles et de procédures propres aux institutions publiques feraient en quelque sorte perdre de vue l'objectif principal poursuivi et contribueraient à « saper » le moral des salariés (GORE, 1993).

Les résultats révèlent enfin que le jugement porté par les salariés sur l'équité de traitement dans le travail réduit de façon significative la probabilité de faire des heures supplémentaires gratuites au sein du privé, tandis que cette variable n'a pas d'effet significatif sur l'offre d'effort supplémentaire des agents de la FPE. Il semble donc que les heures supplémentaires non compensées effectivement réalisées dans le privé et dans la FPE renforcent la perception d'iniquité de la balance investissementbénéfices et contribuent à une dégradation du sentiment de reconnaissance. La théorie d'échange de don que nous avons voulu tester au départ au sein de la FPE n'est donc pas vérifiée ici.

On peut donc supposer qu'une des raisons expliquant l'absence de différence de probabilité dans la réalisation d'heures supplémentaires non compensées entre les deux secteurs provient du fait que ce sur-effort était un comportement existant en début de carrière, mais qui aurait disparu en raison de l'érosion de la motivation intrinsèque présente initialement chez les agents de l'État.

\section{Résultats du modèle de décomposition détaillée}

Dans cette partie, nous cherchons à déterminer dans quelle mesure la différence totale de recours aux heures supplémentaires non compensées entre les deux secteurs est attribuable aux différences de distribution des caractéristiques observables entre les deux populations de salariés. Le tableau 4 présente les résultats de la décomposition de l'écart de probabilité de faire ces heures entre les deux secteurs selon la méthode de R. FAIRLIE (2005). Nous choisissons ici d'interpréter les résultats de la décomposition qui utilisent les coefficients des salariés du secteur privé (colonne 1) comme 
pondération, car nous cherchons à mettre en évidence l'existence d'un comportement spécifique aux agents de la FPE en termes d'heures supplémentaires non compensées. Ainsi, le comportement des salariés du secteur privé est supposé être la situation normale, ce qui sous-entend que les écarts de comportement en termes d'heures supplémentaires non payées des agents de la FPE seraient alors considérés comme du don de travail, lié à une motivation prosociale supérieure.

TABLEAU 4 - Décomposition de Fairlie de l'écart de probabilité de faire des heures supplémentaires non compensées (HSNC) selon le secteur pris comme référence (privé ou public)

\begin{tabular}{|c|c|c|}
\hline Groupe de référence & Privé (1) & Public (2) \\
\hline N Privé & 10154 & 10154 \\
\hline N Public & 949 & 949 \\
\hline $\operatorname{Pr}\left(Y_{j}=1\right)$ si Privé & 0,2345 & 0,2345 \\
\hline $\operatorname{Pr}\left(Y_{i}=1\right)$ si Public & 0,2234 & 0,2234 \\
\hline Différence totale des HSNC & 0,0111 & 0,0111 \\
\hline Part attribuée aux caractéristiques & $-0,0228(-205,4 \%)$ & $-0,0374(-337 \%)$ \\
\hline \multicolumn{3}{|c|}{ Contribution des déterminants à l'écart de probabilité observé } \\
\hline Caractéristiques sociodémographiques & $\begin{array}{c}-0,0157^{* * * *}(68,8 \%) \\
(0,0035)\end{array}$ & $\begin{array}{l}-0,0234^{* * *} \\
(0,01173)\end{array}$ \\
\hline Homme & $\begin{array}{c}-0,0043^{* * * *}(18,8 \%) \\
(0,0016)\end{array}$ & $\begin{array}{l}-0,0014 \\
(0,0051)\end{array}$ \\
\hline Personnes à charge & $\begin{array}{c}-0,0006^{*}(2,6 \%) \\
(0,0003)\end{array}$ & $\begin{array}{l}-0,0007 \\
(0,0009)\end{array}$ \\
\hline Âge & $\begin{array}{c}-0,0069^{* *}(30,2 \%) \\
(0,0027)\end{array}$ & $\begin{array}{c}-0,0188^{* *} \\
(0,0087)\end{array}$ \\
\hline Diplôme & $\begin{array}{c}-0,00900^{* * * *}(39,4 \%) \\
(0,0015)\end{array}$ & $\begin{array}{l}-0,0060 \\
(0,0046)\end{array}$ \\
\hline Technique & $\begin{array}{c}0,0014 \\
(0,0017)\end{array}$ & $\begin{array}{c}0,0036 \\
(0,0055)\end{array}$ \\
\hline Baccalauréat & $\begin{array}{c}-0,0020^{*}(8,8 \%) \\
(0,0011)\end{array}$ & $\begin{array}{l}-0,0051 \\
(0,0040)\end{array}$ \\
\hline Supérieur court & $\begin{array}{c}0,0024 * * *(-10,5 \%) \\
(0,0008)\end{array}$ & $\begin{array}{c}0,0030 \\
(0,0033)\end{array}$ \\
\hline Supérieur long & $\begin{array}{c}-0,0112^{* * *}(49,1 \%) \\
(0,0017)\end{array}$ & $\begin{array}{l}-0,0076 \\
(0,0049)\end{array}$ \\
\hline Ancienneté & $\begin{array}{c}0,0044^{* * *}(-19,29 \%) \\
(0,0022)\end{array}$ & $\begin{array}{c}0,0046 \\
(0,0087)\end{array}$ \\
\hline $1-3$ ans & $\begin{array}{c}0,0016 \\
(0,0011)\end{array}$ & $\begin{array}{l}0,0179^{* * * *} \\
(0,0066)\end{array}$ \\
\hline $3-5$ ans & $\begin{array}{c}0,0012^{*}(-5,2 \%) \\
(0,0006)\end{array}$ & $\begin{array}{l}0,0064^{*} \\
(0,0034)\end{array}$ \\
\hline$>10$ ans & $\begin{array}{c}0,0016 \\
(0,0023)\end{array}$ & $\begin{array}{l}0,0204^{* *} \\
(0,0087)\end{array}$ \\
\hline PCS & $\begin{array}{c}0,0006 \\
(0,0004)\end{array}$ & $\begin{array}{c}0,0006 \\
(0,0031)\end{array}$ \\
\hline Cadre & $\begin{array}{c}-0,0010{ }^{* * * *}(4,4 \%) \\
(0,0003)\end{array}$ & $\begin{array}{l}-0,0021 \\
(0,0018)\end{array}$ \\
\hline Employé-ouvrier & $\begin{array}{c}0,0016^{* * *}(-7 \%) \\
(0,0004)\end{array}$ & $\begin{array}{c}0,0014 \\
(0,0018)\end{array}$ \\
\hline
\end{tabular}




\begin{tabular}{|c|c|c|}
\hline Groupe de référence & Privé (1) & Public (2) \\
\hline Caractéristiques d'emploi & $\begin{array}{c}-0,0071(31,2 \%) \\
(0,0047)\end{array}$ & $\begin{array}{l}-0,0131 \\
(0,0126)\end{array}$ \\
\hline Rapport au temps & $\begin{array}{c}0,0070 \text { *** }(-30,7 \%) \\
(0,0034)\end{array}$ & $\begin{array}{c}0,0097 \\
(0,0081)\end{array}$ \\
\hline Délai & $\begin{array}{c}-0,0057^{* * *}(25 \%) \\
(0,0015)\end{array}$ & $\begin{array}{l}-0,0002 \\
(0,0038)\end{array}$ \\
\hline Objectif & $\begin{array}{c}0,0011^{*}(-4,4 \%) \\
(0,0006)\end{array}$ & $\begin{array}{c}0,0002 \\
(0,0016)\end{array}$ \\
\hline Stress & $\begin{array}{c}0,0000 \\
(0,0002)\end{array}$ & $\begin{array}{c}0,0006 \\
(0,0009)\end{array}$ \\
\hline Heures hebdomadaires & $\begin{array}{c}0,0080^{* * * *}(-35,1 \%) \\
(0,0008)\end{array}$ & $\begin{array}{l}0,0071^{*} \\
(0,0036)\end{array}$ \\
\hline Temps partiel & $\begin{array}{c}-0,0023^{* *}(10,1 \%) \\
(0,0010)\end{array}$ & $\begin{array}{l}-0,0064^{* * *} \\
(0,0030)\end{array}$ \\
\hline Supervision & $\begin{array}{r}-0,0001 \\
(0,0002)\end{array}$ & $\begin{array}{l}-0,0002 \\
(0,0008)\end{array}$ \\
\hline Contrôle & $\begin{array}{r}-0,0001 \\
(0,0004)\end{array}$ & $\begin{array}{l}-0,0002 \\
(0,0011)\end{array}$ \\
\hline Syndicat & $\begin{array}{c}0,0062^{* *}(-27 \%) \\
(0,0028)\end{array}$ & $\begin{array}{l}0,0099^{*} \\
(0,0059)\end{array}$ \\
\hline Effet de signal & $\begin{array}{l}-0,0003 \\
(0,0021)\end{array}$ & $\begin{array}{l}-0,0104 \\
(0,0068)\end{array}$ \\
\hline Opportunité de carrière & $\begin{array}{c}0,0003 \\
(0,0003)\end{array}$ & $\begin{array}{c}0,0001 \\
(0,0007)\end{array}$ \\
\hline Augmentation de salaire & $\begin{array}{l}-0,0019 \\
(0,0013)\end{array}$ & $\begin{array}{l}-0,0048 \\
(0,0042)\end{array}$ \\
\hline Salaire variable & $\begin{array}{c}0,0007 \\
(0,0015)\end{array}$ & $\begin{array}{l}0,0124^{* *} \\
(0,0052)\end{array}$ \\
\hline Log salaire par quinzaine & $\begin{array}{c}0,0006 \\
(0,0005)\end{array}$ & $\begin{array}{c}0,0026 \\
(0,0035)\end{array}$ \\
\hline Organisation du travail et production & $\begin{array}{c}-0,0091^{\text {***** }}(40 \%) \\
(0,0022)\end{array}$ & $\begin{array}{l}-0,0098 \\
(0,0071)\end{array}$ \\
\hline Groupe & $\begin{array}{r}-0,0002 \\
(0,0002)\end{array}$ & $\begin{array}{l}-0,0000 \\
(0,0005)\end{array}$ \\
\hline IU management & $\begin{array}{c}-0,0050 \text { **** }(30 \%) \\
(0,0012)\end{array}$ & $\begin{array}{r}-0,0055 \\
(0,0034)\end{array}$ \\
\hline IU TIC & $\begin{array}{c}-0,0037^{* * * *}(16,2 \%) \\
(0,0013)\end{array}$ & $\begin{array}{l}-0,0036 \\
(0,0049)\end{array}$ \\
\hline Ordinateur portable & $\begin{array}{c}-0,0002 \\
(0,0002\end{array}$ & $\begin{array}{r}-0,0008 \\
(0,0008)\end{array}$ \\
\hline Environnement & $\begin{array}{c}-0,0044^{\text {**** }}(19,3 \%) \\
(0,0010)\end{array}$ & $\begin{array}{l}-0,0029 \\
(0,0032)\end{array}$ \\
\hline Ambiance entre collègues & $\begin{array}{l}-0,0001 \\
(0,0002)\end{array}$ & $\begin{array}{l}-0,0001 \\
(0,0006)\end{array}$ \\
\hline Nouvelle & $\begin{array}{c}-0,0016^{* *}(7 \%) \\
(0,0008)\end{array}$ & $\begin{array}{r}-0,0016 \\
(0,0025)\end{array}$ \\
\hline Reconnaissance & $\begin{array}{c}-0,0038^{* * * *}(16,7 \%) \\
(0,0005)\end{array}$ & $\begin{array}{l}-0,0020 \\
(0,0015)\end{array}$ \\
\hline Zone d'habitation : Paris & $\begin{array}{c}0,0008^{* *}(-3,5 \%) \\
(0,0004)\end{array}$ & $\begin{array}{c}0,0006 \\
(0,0012)\end{array}$ \\
\hline
\end{tabular}

Significativité à : *** $1 \%, * * 5 \%, * 10 \%$.

Lecture : la composante environnementale explique $19,3 \%$ de la différence de probabilité de faire des heures supplémentaires non payées attribuée à l'ensemble des caractéristiques observées entre les deux secteurs $(-0,0044 /-0,0029)$.

Champ : salariés stables ( 1 an d'ancienneté) des unités productives de 20 salariés et plus dans le secteur privé, de 10 salariés et plus dans le secteur public. L'écart-type est reporté entre parenthèses.

Sources : enquête COI 2006 (Insee, Dares); COI-FP 2006 (Dares, DGAFP, CEE). 
Les résultats montrent que plus du double de l'écart de probabilité de faire des heures supplémentaires non compensées entre les deux secteurs (205,4\% précisément) est expliqué par des différences dans la distribution des caractéristiques observables, mais aussi que cette contribution s'avère négative. Cela signifie que si les agents de la FPE étaient rendus identiques aux salariés du privé en termes de caractéristiques individuelles et d'emploi observables, l'écart de probabilité de faire ces heures supplémentaires augmenterait de 205,4 \% ; c'est-à-dire que les agents de la FPE auraient une probabilité nettement plus faible de fournir ces heures. Nous pouvons supposer que la façon dont sont répartis les individus ainsi que l'application de méthodes de gestion de la main-d'œuvre et d'organisation du travail différentes au sein de chaque secteur correspondent ainsi bien à la répartition permettant de favoriser le recours à ces heures au sein des deux secteurs. Par ailleurs, le fait d'avoir une partie expliquée négative signifie que la partie inexpliquée (qui inclut la motivation intrinsèque) tend à expliquer une part très importante de la différence de probabilité de faire des heures supplémentaires non payées.

En plus de la contribution totale des variables explicatives à l'écart de probabilité observé, nous examinons à des fins d'analyse les contributions de chacune des différentes variables explicatives et celles de groupements de variables par catégories d'explications théoriques.

Ainsi, parmi l'ensemble des variables introduites dans le modèle, celles qui relèvent des caractéristiques sociodémographiques expliquent le plus les différences de probabilité de faire des heures supplémentaires gratuites entre les deux secteurs, puisque cette composante représente $68 \%$ de l'écart attribuable aux caractéristiques observables (estimation significative à $1 \%$ ). Si les caractéristiques des agents de la FPE se rapprochaient de celles des salariés du secteur privé, qui sont plus souvent des hommes, plus jeunes et moins diplômés, cela augmenterait l'écart de probabilité existant ${ }^{28}$.

Parmi les caractéristiques liées à l'organisation du travail et de la production, l'intensité d'utilisation des outils de management et des technologies de l'information et de la communication (TIC) contribue globalement à réduire l'écart de probabilité de faire des heures non compensées entre les deux secteurs. Autrement dit, si l'intensité d'utilisation des outils managériaux mis en œuvre dans le secteur privé était importée de façon identique dans la FPE, alors l'écart de probabilité observé augmenterait. En effet, les méthodes de management issues des pratiques exercées dans le secteur concurrentiel ne sont pas toujours adaptées, ni bien perçues, lorsqu'elles sont appliquées dans le secteur public. Nous retrouvons donc ici un résultat cohérent avec la théorie de l'éviction de motivation intrinsèque évoquée précédemment (FREY, 1997 ; BéNABOU, TiRole, 2003).

28. En raison d'une répartition très inégale entre les catégories « employés » et « ouvriers » au sein des deux secteurs, nous choisissons de créer un seul indicateur regroupant ces deux catégories socioprofessionnelles pour estimer notre modèle. En effet, le plus grand nombre d'ouvriers dans le secteur privé par rapport à la FPE (respectivement présents à hauteur de 37,4\% et 8,6 \%) ainsi que le nombre supérieur d'employés dans la FPE par rapport au privé (respectivement $45 \%$ contre 14,4\%) auraient entraîné un biais dans l'écart de probabilité observé, voir tableau B en annexe. 
Par ailleurs, la différence de taux de participation à une union syndicale entre les deux secteurs (qui est plus élevé dans la FPE) contribue positivement à l'écart de probabilité de faire des heures supplémentaires non payées. Ainsi, les agents de la FPE auraient une probabilité relativement plus élevée de faire ces heures par rapport aux salariés du privé s'ils étaient moins syndiqués. Ces résultats s'opposent à ceux trouvés dans l'étude de M. Veliziotis (2010), qui montrent que le don de travail est plus susceptible de se matérialiser parmi les membres de syndicats lorsque ceux-ci exercent dans des secteurs fournissant des services sociaux et non marchands. Ils font davantage preuve d'une attitude prosociale en raison d'une éthique prosociale spécifique qui les caractérise. Il se peut que les changements organisationnels initiés depuis 2003 au sein de la fonction publique aient renforcé la vigilance des syndicats, qui exercent alors plus ardemment leur rôle de protection contre l'exploitation de la main-d'œuvre en négociant plus âprement la rémunération de ces heures ${ }^{29}$.

Parmi les caractéristiques associées à la gestion du temps de travail, l'imposition moins fréquente de délais dans la FPE (tableau B en annexe) contribue à réduire l'écart de probabilité de faire des heures supplémentaires non payées. Cela suggère que si les agents de la FPE étaient assujettis aux mêmes contraintes de gestion de leur temps de travail que les salariés du secteur privé, leur probabilité d'offrir ces heures supplémentaires serait réduite.

En outre, le poids des variables introduites pour capter la qualité de l'environnement de travail des salariés contribue négativement à l'écart de probabilité observé de faire ces heures entre les deux secteurs. C'est le cas pour l'intérêt de l'emploi, mesuré par le fait d'indiquer apprendre régulièrement des choses nouvelles, ainsi que pour le constat d'une bonne ambiance entre collègues, pourtant plus fréquents au sein de la FPE. Les résultats révèlent également que si les agents de la FPE se considéraient davantage traités à leur juste valeur (autrement dit, si étaient pris en compte ce qu'ils apportent dans l'organisation et ce qu'ils reçoivent en retour), cet écart entre les deux secteurs se creuserait. La théorie du don - contre-don, qui stipule que les salariés qui se sentent bien traités en termes de conditions de travail peuvent alors augmenter leur niveau d'effort (AKERLOF, 1982), n'est ici pas vérifiée.

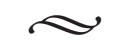

Cette étude révèle que la motivation prosociale des agents de la fonction publique d'État ne s'exprime pas à travers un surplus d'effort en termes d'heures supplémentaires non compensées par rapport aux salariés du secteur privé, toutes choses égales par ailleurs. Toutefois, ce comportement ne s'explique pas par les mêmes facteurs déterminants au sein des deux secteurs, ce qui laisse penser que les sources de motivation pour l'effort sont différentes entre les deux types de travailleurs.

29. Il se peut également que les syndicalistes soient plus attentifs à ne pas travailler au-delà de ce qu'ils sont rémunérés et ce d'autant plus que leurs engagements syndicaux, s'ajoutant à leur activité professionnelle, sont susceptibles d'empiéter sur leur temps personnel. 
L'analyse des différents déterminants du comportement d'offre d'heures supplémentaires a en effet montré que l'introduction par l'employeur de mécanismes incitatifs standard (menace de licenciement ou rémunération à la performance) exerce un effet positif et significatif sur l'offre d'effort uniquement pour les salariés du secteur privé. Tandis que dans le secteur public, l'utilisation de certains de ces mécanismes incitatifs (comme la variabilité des salaires) joue un rôle inverse, étant négativement corrélée avec l'offre d'effort gratuit de la part des agents de l'État.

Les résultats de notre étude montrent que la répartition des individus et le choix des éléments organisationnels et incitatifs mis en œuvre au sein de chaque secteur favorisent effectivement l'obtention d'une offre d'efforts supplémentaires, et que l'homogénéisation éventuelle des caractéristiques d'emploi (les méthodes de management et d'organisation du travail observées dans le secteur privé seraient importées au sein de l'administration d'État) contribuerait à réduire le comportement d'offre d'heures supplémentaires des agents publics par rapport aux salariés du secteur privé. Certaines formes de mobilisation de la main-d'œuvre dans le secteur public sont visiblement susceptibles de réduire le potentiel lié à la motivation prosociale de leurs agents.

Les réformes récentes, inspirées du nouveau management public, pourraient ainsi jouer un rôle dans cette diminution. En effet, l'offre d'heures supplémentaires révèle des sources de motivation pour l'effort propres aux salariés de chaque secteur. Aussi, si les agents publics ne font pas montre d'un recours à l'effort plus élevé que les salariés du privé, cela peut être lié aux nouveaux modes de gestion de la main-d'œuvre engagés à l'occasion de ces réformes. La motivation prosociale, initialement présente chez les agents de la FPE, pourrait avoir disparu, ce qui serait le signal d'un rejet des méthodes de gestion de la main-d'œuvre mal adaptées au secteur public. Ce comportement, apparemment anormal d'un point de vue de la théorie traditionnelle, peut être expliqué par la théorie de l'éviction de la motivation intrinsèque (FREY, 1997) selon laquelle les récompenses monétaires et le contrôle exercé sur les travailleurs motivés intrinsèquement risquent d'évincer la motivation initialement présente et de diminuer ainsi la performance.

Ainsi, notre étude ne contribue pas uniquement à la littérature sur la mise en évidence d'une motivation spécifique des agents du secteur public en France, mais peut aussi avoir des implications en termes de management des ressources humaines. Le secteur public est soumis depuis quelques années à des réorganisations profondes de ses outils de gestion du personnel, dont l'efficacité demeure encore incertaine. En effet, l'introduction de méthodes de gestion de la main-d'œuvre directement importées du secteur marchand, comme la promotion ou la rémunération au mérite, revient à ignorer la spécificité des formes de motivation qui animent les agents de l'État et peut s'avérer non pertinente.

Pour confirmer la présence de motivations différentes dans les secteurs public et privé, et l'efficacité relative de différentes formes d'incitation, il conviendrait toutefois de corriger certaines limites de notre étude. Une première préoccupation provient du caractère qualitatif de notre mesure de l'effort. Il n'est pas possible de quantifier le nombre réel d'heures supplémentaires non compensées réalisées par les salariés. Aussi, lorsque 
les travailleurs réalisent dix heures supplémentaires de travail et ne sont rémunérés que pour cinq, on ne peut savoir s'ils vont les déclarer en totalité ou en partie. Cela donne donc une dimension subjective et ambiguë à la variable expliquée. De la même manière, les données de l'enquête ne nous permettent pas de contrôler les différents domaines d'activité des secteurs public et privé. À des fins d'analyses plus fines, il serait intéressant de comparer des secteurs à mission strictement identique, quelle que soit la forme institutionnelle de l'institution pourvoyeuse du service, comme le secteur hospitalier ${ }^{30}$. Une telle perspective nous permettrait de tester une version consolidée de la théorie du don de travail reposant uniquement sur l'hypothèse de différence institutionnelle de l'employeur.

\section{BibliogRAPHIE}

AKerLof G. A. (1982), "Labor Contracts as Partial Gift Exchange", The Quarterly Journal of Economics, vol. 97, n ${ }^{\circ}$ 4, pp. 543-569.

Anger S. (2008), "Overtime Work as a Signaling Device", Scottish Journal of Political Economy, vol. 55, n² 2, pp. 167-189.

ANGER S. (2011), "The Cyclicality of Effective Wages within Employer-Employee Matches in a Rigid Labor Market", Labour Economics, vol. 18, n 6, pp. 786-797.

AUDIER F. (2000), « La transmission du statut dans la fonction publique », Économie et statistique, n ${ }^{\circ} 337-338$, pp. 121-133.

Bell D. N. F., HART R. A. (1999), “Unpaid Work”, Economica, vol. 66, nº 262, pp. 271-290.

Bénabou R., Tirole J. (2003), "Intrinsic and Extrinsic Motivation", The Review of Economic Studies, vol. 70, $\mathrm{n}^{\circ} 3$, pp. 489-520.

Besley T., GHATAK M. (2005), "Competition and Incentives with Motivated Agents", American Economic Review, vol. 95, $\mathrm{n}^{\circ} 3$, pp. 616-636.

Bigi M., Greenan N., Hamon-Cholet S., Lanfranchi J. (2012), « Changements organisationnels et évolution du vécu au travail des salariés : une comparaison entre secteur privé et fonction publique d'État », Rapport de recherche, $\mathrm{n}^{\circ} 75$, Centre d'études de l'emploi.

BLinder A. S. (1973), "Wage Discrimination: Reduced Form and Structural Estimates", The Journal of Human Resources, vol. 8, $\mathrm{n}^{\circ}$ 4, pp. 436-455.

Bratti M., Staffolani S. (2007), "Effort-Based Career Opportunities and Working Time", International Journal of Manpower, vol. 28, n 6, pp. 489-512.

Buurman M., Delfgaauw J., Dur R., Van den Bossche S. (2012), "Public Sector Employees: Risk Averse and Altruistic?", Journal of Economic Behavior \& Organization, vol. 83, n 3 , pp. 279-291.

DECI E. L. (1971), "Effects of Externally Mediated Rewards on Intrinsic Motivation”, Journal of Personality and Social Psychology, vol. 18, n ${ }^{\circ}$ 1, pp. 105-115.

30. En raison de la non-disponibilité de la variable des salaires dans les hôpitaux publics, indispensable dans notre étude, nous n'avons pu exploiter le champ d'extension de l'enquête COI-H. 
Delfgaauw J., Dur R. (2008), “Incentives and Workers' Motivation in the Public Sector”, The Economic Journal, vol. 118, n ${ }^{\circ}$ 525, pp. 171-191.

Demoly E. (2011), « Heures supplémentaires et rachat de jours de congé : les dispositifs d'allongement du temps de travail vus par les salariés », Dares analyses, $\mathrm{n}^{\circ} 54$.

Engellandt A., Riphahn R. T. (2005), “Temporary Contracts and Employee Effort”, Labour Economics, vol. 12, n 3, pp. 281-299.

FAIRLIE R. W. (2005), “An Extension of the Blinder-Oaxaca Decomposition Technique to Logit and Probit Models", Journal of Economic and Social Measurement, vol. 30, n ${ }^{\circ}$ 4, pp. 305-316.

FRANÇOIS P. (2000), “Public Service Motivation' as an Argument for Government Provision”, Journal of Public Economics, vol. 78, n ${ }^{\circ} 3$, pp. 275-299.

FrançoIs P. (2007), "Making a Difference”, The Rand Journal of Economics, vol. 38, n 3, pp. 714-732.

François P., Vlassopoulos M. (2008), "Pro-Social Motivation and the Delivery of Social Services”, Cesifo Economic Studies, vol. 54, n 1, pp. 22-54.

FREY B. S. (1997), "On the Relationship between Intrinsic and Extrinsic Work Motivation”, International Journal of Industrial Organization, vol. 15, n 4, pp. 427-439.

Gore A. (1993), From Red Tape to Results: Creating a Government That Works Better and Costs Less. Report of the National Performance Review, Washington, US Government Printing Office.

Grant A. M. (2008), “The Significance of Task Significance: Job Performance Effects, Relational Mechanisms, and Boundary Conditions", Journal of Applied Psychology, vol. 93, n 1, pp. 108-124.

GreEnAN N., HAMON-ChOlEt S., LANFRANCHI J. (2016), « Le processus de changement dans les secteurs privé et public : une comparaison », in Greenan N., Hamon-Cholet S., Ughetto P., Salariés du public, salariés du privé face aux changements, Paris, L'Harmattan, pp. 25-63.

Gregg P., Grout P. A., Ratcliffe A., Smith S., Windmeijer F. (2011), "How Important is Pro-Social Behaviour in the Delivery of Public Services?", Journal of Public Economics, vol. 95, n ${ }^{\circ} 7-8$, pp. 758-766.

HÜBLER O. (2000), “All Goes Faster but Lasts Longer: Computer Use and Overtime Work”, Ifo Studien, vol. 46, $\mathrm{n}^{\circ}$ 2, pp. 49-271.

IoAnnides A., Oxouzi E., Mavroudeas S. (2014), "All Work and No... Pay? Unpaid Overtime in Greece: Determining Factors and Theoretical Explanations", Industrial Relations Journal, vol. $45, \mathrm{n}^{\mathrm{o}} 1$, pp. 39-55.

LANFRANCHI J., NARCY M. (2006), "Wages and Effort in the French For-Profit and Nonprofit Sectors: Labor Donation Theory Revisited”, Working Papers Ermes, n 06-20.

LANFranchi J., Pekovic S. (2014), "How Green is my Firm? Workers’ Attitudes and Behaviors towards Job in Environmentally-Related Firms", Ecological Economics, vol. 100, pp. 16-29.

L'Horty Y. (2016), Les Discriminations dans l'accès à l'emploi public, rapport au Premier ministre, Paris.

Lucifora C., Meurs D. (2006), "The Public Sector Pay Gap in France, Great Britain and Italy”, The Review of Income and Wealth, vol. 52, n 1, pp. 43-59. 
McKenZie T., Rutherford A. C. (2017), "Career Concerns Versus Shared Values: An Empirical Investigation”, German Journal of Human Resource Management, vol. 31, n 2, pp. 162-184. VAN DER MEer P. H., Wielers R. (2015), “Unpaid Overtime in the Netherlands: Forward- or Backward-Looking Incentives?”, International Journal of Manpower, vol. 36, n 3, pp. 254-270.

Melnik E., Guillemot D. (2010), « Vers une convergence du management public-privé ? Une revue de littérature économique », Revue française d'économie, vol. 25, n² 2, pp. 167-225.

MeYer A., WALlette M. (2005), "Absence of Absenteeism and Overtime Work - Signalling Factors for Temporary Workers?”, Economics Department Working Paper, n ${ }^{\circ}$ 2005-15, Lund University.

Mintzberg H. (1983), Power in and around Organizations, Englewood Cliffs, London, Prentice-Hall.

Monfardini C., Radice R. (2008), “Testing Exogeneity in the Bivariate Probit Model: A Monte Carlo Study", Oxford Bulletin of Economics and Statistics, vol. 70, n 2, pp. 271-282.

Moynihan D. P., Pandey S. K. (2007), “The Role of Organizations in Fostering Public Service Motivation", Public Administration Review, vol. 67, n 1, pp. 40-53.

NARCY M. (2013a), « Le travail associatif : des salariés intrinsèquement motivés », in Hély M., Simonet M. (dir.), Le Travail associatif, Nanterre, Presses universitaires de Paris Ouest, pp. 19-32.

NARCy M. (2013b), La Contribution des méthodes de décomposition détaillée à l'évaluation des inégalités, Présentation, École thématique sur l'évaluation des politiques publiques, Aussois (colloque).

Nyborg K., Zhang T. (2013), “Is Corporate Social Responsibility Associated with Lower Wages?", Environmental and Resource Economics, vol. 55, n 1, pp. 107-117.

OAXACA R. (1973), "Male-Female Wage Differentials in Urban Labor Markets”, International Economic Review, vol. 14, n 3, pp. 693-709.

PAnnenberg M. (2005), "Long Term Effects of Unpaid Overtime. Evidence for West Germany", Scottish Journal of Political Economy, vol. 52, n 2, pp. 177-193.

Perry J. L. (2000), “Bringing Society In: Toward a Theory of Public-Service Motivation”, Journal of Public Administration Research and Theory, vol. 10, n 2, pp. 471-488.

Perry J. L., Hondeghem A., Wise L. R. (2010), "Revisiting the Motivational Bases of Public Service: Twenty Years of Research and an Agenda for the Future", Public Administration Review, vol. 70, n 5, pp. 681-690.

Preston A. E. (1989), "The Nonprofit Worker in a For-Profit World", Journal of Labor Economics, vol. 7, $\mathrm{n}^{\circ}$ 4, pp. 438-463.

Veliziotis M. (2010), “Trade Unions and Unpaid Overtime in Britain”, ISER Working Paper Series, $\mathrm{n}^{\circ} 2010-43$.

Wanger S., Weigand R., Zapf I. (2016), "Measuring Hours Worked in Germany. Contents, Data and Methodological Essentials of the IAB Working Time Measurement Concept", Journal for Labour Market Research, vol. 49, n 3, pp. 213-238. 
Motivation prosociale et don de travail :

une comparaison entre le secteur privé et la fonction publique d'État en France

\section{Annexe}

\section{TABLEAU A - Description des variables utilisées}

\begin{tabular}{|c|c|}
\hline Nom de la variable & Description \\
\hline HSNC & Compensation en salaire ou en repos en cas de dépassement horaire \\
\hline Public & Salarié appartenant à la fonction publique d'État \\
\hline Homme & Genre du salarié \\
\hline$\hat{A} g e$ & Âge du salarié (variable continue) \\
\hline Personnes à charge & Nombre de personnes à charge du salarié ou de son conjoint vivant au foyer \\
\hline Primaire & Niveau d'études le plus élevé obtenu (enseignement du $1^{\mathrm{er}}$ degré) \\
\hline Technique & $\begin{array}{l}\text { Niveau d'études le plus élevé obtenu (certificat d'aptitude professionnelle [CAP], } \\
\text { brevet d'études professionnelles [BEP]) }\end{array}$ \\
\hline Baccalauréat & Niveau d'études le plus élevé obtenu \\
\hline Supérieur court & Niveau d'études le plus élevé obtenu : $1^{\mathrm{er}}$ et $2^{\mathrm{e}}$ cycles \\
\hline Supérieur long & Niveau d'études le plus élevé obtenu : $3^{\mathrm{e}}$ cycle et grandes écoles \\
\hline $1-3$ ans & Ancienneté dans l'entreprise \\
\hline 3-5 ans & Ancienneté dans l'entreprise \\
\hline $5-10$ ans & Ancienneté dans l'entreprise \\
\hline$<10$ ans & Ancienneté dans l'entreprise \\
\hline Ouvrier & Groupe socioprofessionnel du salarié : ouvrier \\
\hline Employé & Groupe socioprofessionnel du salarié : employé \\
\hline Profession intermédiaire & Groupe socioprofessionnel du salarié : profession intermédiaire \\
\hline Cadre & Groupe socioprofessionnel du salarié : cadre \\
\hline Opportunité de carrière & $\begin{array}{l}\text { L'évaluation de votre travail de l'année } 2005 \text { a eu ou va avoir des conséquences } \\
\text { concrètes sur votre carrière }\end{array}$ \\
\hline Emploi risqué & Risque de perte d'emploi dans l'année qui vient \\
\hline Augmentation de salaire & $\begin{array}{l}\text { L'évaluation de votre travail de l'année } 2005 \text { a eu ou va avoir des conséquences } \\
\text { concrètes sur votre rémunération }\end{array}$ \\
\hline Salaire variable & Une partie ou la totalité du salaire est variable \\
\hline Salaire par quinzaine & Salaire par quinzaine du contrat de travail $^{1}$ \\
\hline Temps partiel & Travailler à temps partiel \\
\hline Heures hebdomadaires & Nombre d'heures de travail effectuées par semaine \\
\hline Groupe & $\begin{array}{l}\text { Faire actuellement partie d'un groupe de travail de type groupe de projet, } \\
\text { groupe de pilotage }\end{array}$ \\
\hline Ordinateur portable & Avoir un ordinateur portable à son domicile \\
\hline Syndicat & Être adhérent ou sympathisant d'une organisation syndicale de salariés \\
\hline IU management & Indicateur d'intensité d'utilisation des outils managériaux \\
\hline IU TIC & Indicateur d'intensité d'utilisation des outils technologiques \\
\hline Délai & Rythme de travail imposé par des normes ou des délais \\
\hline Objectif & Devoir atteindre des objectifs précis \\
\hline Stress & Impression de ne pas pouvoir faire face ou de se sentir débordé \\
\hline Ambiance entre collègues & Bonne ambiance de travail entre collègues \\
\hline Reconnaissance & $\begin{array}{l}\text { Votre travail est-il reconnu à sa juste valeur quand vous faites le bilan de ce que vous } \\
\text { apportez dans votre entreprise et des bénéfices que vous en retirez? }\end{array}$ \\
\hline Nouvelle & Votre travail vous permet-il d'apprendre des nouvelles choses? \\
\hline Supervision & Exercice hiérarchique : avoir des salariés sous ses ordres \\
\hline Contrôle & Votre travail est contrôlé par un supérieur hiérarchique ou une direction du service \\
\hline Mère française & Avoir une mère de nationalité française \\
\hline Père ou mère professeur & Avoir au moins l'un de ses parents professeur \\
\hline
\end{tabular}

1. La variable du salaire est renseignée pour les 949 individus de la FPE et pour 10154 individus du secteur privé. Il s'agit d'un salaire net par quinzaine calculé en fonction du salaire annuel et de la durée de paie. Dans les équations, cette variable correspond au logarithme du salaire horaire net par quinzaine. 
TABlEAU B - Statistiques descriptives

\begin{tabular}{|c|c|c|c|}
\hline Secteurs institutionnels (\%) & Privé & Privé restreint & FPE \\
\hline HSNC & 23,97 & 26,40 & 22,29 \\
\hline \multicolumn{4}{|l|}{ Caractéristiques individuelles } \\
\hline Homme & 63,39 & 62,10 & 45,00 \\
\hline Âge & 41,36 & 40,32 & 46,14 \\
\hline Personnes à charge & 1,11 & 1,07 & 1,04 \\
\hline \multicolumn{4}{|l|}{ Diplôme } \\
\hline Primaire & 11,00 & 10,29 & 9,67 \\
\hline Technique & 34,25 & 18,67 & 24,07 \\
\hline Baccalauréat & 17,25 & 15,44 & 23,65 \\
\hline Supérieur court & 19,58 & 22,90 & 19,03 \\
\hline Supérieur long & 17,90 & 32,67 & 23,55 \\
\hline \multicolumn{4}{|l|}{ Ancienneté } \\
\hline $1-3$ ans & 10,55 & 15,88 & 3,15 \\
\hline $3-5$ ans & 11,22 & 15,30 & 7,25 \\
\hline $5-10$ ans & 25,94 & 31,76 & 15,77 \\
\hline$>10$ ans & 52,28 & 37,05 & 73,81 \\
\hline \multicolumn{4}{|l|}{ PCS } \\
\hline Cadre & 19,04 & 32,43 & 19,13 \\
\hline Profession intermédiaire & 29,01 & 25,98 & 26,91 \\
\hline Employé & 14,89 & 21,51 & 45,32 \\
\hline Ouvrier & 37,04 & 20,06 & 8,60 \\
\hline \multicolumn{4}{|l|}{ Type de contrat } \\
\hline Emploi sans limite de durée ${ }^{1}$ & 97,82 & 96,58 & 97,69 \\
\hline dont temps complet & 91,89 & 86,03 & 86,54 \\
\hline dont temps partiel & 5,93 & 10,55 & 11,15 \\
\hline Emploi à durée limitée & 2,17 & 3,43 & 2,31 \\
\hline
\end{tabular}

\section{Caractéristiques d'emploi}

\section{Rapport au temps}

Délai

Objectif

Stress

Heures hebdomadaires

Contrat à temps partiel

Supervision

Contrôle

Syndicat

54,36

54,36

63,56

49,82

38,14

6,49

32,29

33,85

15,09

\section{Effet de signal}

Opportunité de carrière

Emploi risqué

Augmentation de salaire

Salaire variable

Salaire par quinzaine (en euros)

\section{Autre organisation du travail}

Groupe

IU management

IU TIC

Ordinateur portable

\section{7,67}

10,18

27,64

39,11

1079,32

20,30

41,91

52,91

19,62
47,73

56,11

48,55

37,06

13,76

31,28

72,04

13,33

29,64

10,77

28,72

32,62

1171,26

24,10

23,18

50,17

29,45
33,12

55,41

50,05

37,20

12,09

29,23

33,33

38,27

34,27

0,10

17,24

21,34

979,87 
Motivation prosociale et don de travail :

une comparaison entre le secteur privé et la fonction publique d'État en France

\begin{tabular}{lccc}
\hline \multicolumn{1}{c}{ Secteurs institutionnels (\%) } & Privé & Privé restreint & FPE \\
\hline Environnement & & & 81,07 \\
Ambiance entre collègues & 79,20 & 77,04 & 81,59 \\
Nouvelle & 73,82 & 74,15 & 38,80 \\
Reconnaissance & 44,88 & 44,70 & 13,56 \\
Zone d'habitation : Paris & 16,11 & 32,05 & 8,94 \\
\hline Instruments & & & 89,27 \\
Père ou mère professeur & 5,42 & 8,51 & 86,10 \\
Mère française & 83,26 & 76,56 & 951 \\
Père français & 82,00 & 78,79 & 3398 \\
\hline Observations & 11731 & 3 & \\
\hline
\end{tabular}

1. Cette variable ne permet pas de distinguer si les agents de la FPE sont fonctionnaires ou contractuels puisque le libellé complet des propositions faites aux travailleurs des deux secteurs est le suivant : « Emploi sans limite de durée : CDI, titulaire de la fonction publique à temps partiel » ou « Emploi sans limite de durée : CDI, titulaire de la fonction publique à temps complet ».

Significativité à : *** $1 \%, * * 5 \%, * 10 \%$.

Champ : salariés stables (1 an d'ancienneté) des unités productives de 20 salariés et plus dans le secteur privé, de 10 salariés et plus dans le secteur public.

Sources : enquête COI 2006 (Insee, Dares); COI-FP 2006 (Dares, DGAFP, CEE). 
